\title{
A systematic assessment of current genome-scale metabolic reconstruction tools
}

\author{
Sebastián N. Mendoza', Brett G. Olivier ${ }^{1,2}$, Douwe Molenaar ${ }^{1}$ and Bas Teusink ${ }^{1 *}$ (D)
}

\begin{abstract}
Background: Several genome-scale metabolic reconstruction software platforms have been developed and are being continuously updated. These tools have been widely applied to reconstruct metabolic models for hundreds of microorganisms ranging from important human pathogens to species of industrial relevance. However, these platforms, as yet, have not been systematically evaluated with respect to software quality, best potential uses and intrinsic capacity to generate high-quality, genome-scale metabolic models. It is therefore unclear for potential users which tool best fits the purpose of their research.

Results: In this work, we perform a systematic assessment of current genome-scale reconstruction software platforms. To meet our goal, we first define a list of features for assessing software quality related to genome-scale reconstruction. Subsequently, we use the feature list to evaluate the performance of each tool. To assess the similarity of the draft reconstructions to high-quality models, we compare each tool's output networks with that of the high-quality, manually curated, models of Lactobacillus plantarum and Bordetella pertussis, representatives of gram-positive and gram-negative bacteria, respectively. We additionally compare draft reconstructions with a model of Pseudomonas putida to further confirm our findings. We show that none of the tools outperforms the others in all the defined features.
\end{abstract}

Conclusions: Model builders should carefully choose a tool (or combinations of tools) depending on the intended use of the metabolic model. They can use this benchmark study as a guide to select the best tool for their research. Finally, developers can also benefit from this evaluation by getting feedback to improve their software.

Keywords: Genome-scale metabolic reconstruction, Systematic evaluation, Genome-scale metabolic models, Bordetella pertussis, Lactobacillus plantarum, Pseudomonas putida

\section{Background}

Genome-scale metabolic models (GSMMs) have been a successful tool in Systems Biology during the last decades $[1,2]$, largely due to the wide range of areas for which the scientific community has found an application. GSMMs, for example, predict cellular behavior under different biological conditions, or can be used to design drug targets for important pathogens; they help to design improved strains through metabolic engineering strategies or to predict metabolic interactions in microbial communities; they have been used to study

\footnotetext{
*Correspondence: b.teusink@vu.nl

'Systems Bioinformatics, AIMMS, Vrije Universiteit Amsterdam, Amsterdam,

The Netherlands

Full list of author information is available at the end of the article
}

evolutionary processes or to give a rationale to lab experiments (see excellent reviews $[3,4]$ ).

The reconstruction process that forms the basis of a GSMM is very time-consuming. Usually, this process starts with the annotation of a genome and the prediction of candidate metabolic functions at a genome-scale. The draft reconstruction is then refined by the user in an iterative manner through an exhaustive review of each reaction, metabolite, and gene in the network. After curation, the genome-scale metabolic reconstruction is transformed into a mathematical structure, an objective function is given, constraints are set to account for specific media conditions and the resulting GSMM is evaluated to try to reproduce the experimental data. This iterative process of manual refinement is the limiting 
step of the whole process because it continues until the GSMM achieves the desired performance determined by the model builder. Hundreds of GSMMs have been reconstructed using this procedure, for which protocols have been described [5] and reviews are available [6, 7].

Several genome-scale reconstruction tools have been developed over the last 15 years to assist researchers in the reconstruction process $[8,9]$. These tools are designed to speed up such process by automating several tasks that otherwise should be performed manually, such as draft network generation or gap-filling, and/or by providing useful information to the user to curate the reconstruction. There has been an outstanding increase in the number of new tools for genome-scale reconstruction which reflects the increasing interest to create highquality GSMMs [10]. Consequently, there is a need for a systematic assessment of the performance of these tools, as many researchers are uncertain which tool to choose when they want to reconstruct their favorite organisms.

In this work, we installed and applied the most promising genome-scale reconstruction tools to provide a systematic evaluation of their performance and outputs. With each tool we reconstructed draft networks for Lactobacillus plantarum [11] and Bordetella pertussis [12], representatives of gram-positive and gram-negative bacteria, respectively, and for which high-quality GSMMs already exist. We used high-quality manually curated GSMMs as a benchmark to assess the features of the tool-generated draft models. In addition, we also reconstructed draft networks for Pseudomonas putida to confirm our findings.

\section{Current state of genome-scale reconstruction tools}

Here, we provide a brief description of the current reconstruction tools (see also Additional file 1: Table S1).

\section{AutoKEGGRec (2018)}

AutoKEEGRec [13] is an easy-to-use automated tool that uses the KEGG databases to create draft genomescale models for any microorganism in that database. It runs in MATLAB and is compatible with COBRA Toolbox v3 [14]. One of the advantages of this tool is that multiple queries (microorganisms) can be processed in one run making it appropriate for cases where several microorganisms need to be reconstructed. The main limitation of this tool, which is directly related to the use of the KEGG database, is the lack of a biomass reaction, transport and exchange reactions in the draft genomescale models.

AuReMe (2018)

AuReMe [15] (Automatic Reconstruction of Metabolic Models) is a workspace that ensures good traceability of the whole reconstruction process, a feature that makes this tool unique. A Docker image is available for AuReMe, so users are easily able to run AuReMe in any platform without having to pre-install required packages (Windows, Linux or Mac). AuReMe creates GSMMs with a template-based algorithm [16] but it is also designed to incorporate information from different databases such as MetaCyc [17] and BIGG [18].

\section{CarveMe (2018)}

CarveMe [19] is a command-line python-based tool designed to create GSMMs, ready to use for Flux Balance Analysis (FBA), in just a few minutes. Its unique topdown approach involves the creation of models from a BIGG-based manually curated universal template. The implementation of its own gap-filling algorithm allows this tool to prioritize the incorporation into the network of reactions with higher genetic evidence. The authors of this tool showed that the performance of the generated models is similar to the manually curated models.

\section{MetaDraft (2018)}

MetaDraft $[20,21]$ is a Python-based user-friendly software designed to create GSMMs from previously manually curated ones. It contains in its internal database BIGG models ready to be used as templates although any other model can be used as a template. Users can define a specific order of templates in order to prioritize the incorporation of information related to reactions if there is a reaction match in two or more templates. One of the advantages of Metadraft is that it supports the latest features of the current SBML standards, i.e., SBML Level 3 [22] including the FBC Version 2 [23] and Groups packages [24].

\section{RAVEN version 2 (2018)}

RAVEN [25] (Reconstruction, Analysis and Visualization of Metabolic Networks) is a tool for genome-scale metabolic reconstruction and curation that runs in MATLAB is compatible with COBRA Toolbox v3 [14]. In contrast to the first version which only allowed reconstruction using the KEGG database [26], this evaluated version also allows the novo reconstruction of GSMMs using MetaCyc and from template models. Additionally, algorithms to merge network from both databases are provided inside RAVEN. The addition of MetaCyc allows the incorporation of transporters and spontaneous reactions to the reconstructed networks.

\section{ModelSEED version 2.2 (2018)}

ModelSEED [27] is a web resource for genome-scale reconstruction and analysis. This tool allows the creation of GSMMs, not only for microorganisms but also for plants. The first step of its pipeline for genome-scale reconstruction is the genome annotation which is 
performed by RAST [28]. Users can select or even create a medium to be used for gap-filling. In contrast to the first version, the second version allows the creation of models in less than 10 min (including annotation) and it provides aliases/synonyms of reactions and metabolites in other databases.

\section{Pathway Tools version 22.0 (2018)}

Pathway tools [29] is a software environment that supports the creation and curation of organism-specific databases. One of the most useful features is that users can interactively explore, visualize and edit different components of the created databases such as genes, operons, enzymes (including transporters), metabolites, reactions, and pathways. Also, visualization of the whole network is possible by using Cellular Overview diagrams, in which experimental data such as gene expression can be mapped using different colors depending on the expression level.

\section{Merlin version 3.8 (2018)}

Merlin [30] is a java application for genome-scale reconstruction based on the KEGG database. One of the most useful resources of Merlin is the re-annotation of genomes through the online service of BLAST (EBI) or HMMER. Several parameters in the annotation algorithms such as the expected value threshold and the maximum number of hits can be changed by the user if required, which makes this tool very flexible. The interface allows to compare gene function agreement between the annotation and UniProt providing information to the user for manual curation.

\section{Kbase (2018)}

Kbase [31] (The US Department of Energy Systems Biology Knowledgebase) is an open-source software that allows, among a variety of functions, the reconstruction, and analysis of microbes, plants, and communities. Kbase is a platform that integrates several tasks such as annotation, reconstruction, curation, and modeling, making suitable for the whole process of reconstruction. One of the unique features of this software is the use of narratives which are tutorials where users can interactively learn particular topics and reproduce previous results.

\section{CoReCO (2014)}

CoReCo [32] (Comparative Reconstruction) is a novel approach for the simultaneous reconstruction of multiple related species. The pipeline of CoReCo includes two steps: First, it finds proteins homologous to the input set of protein-coding sequences for each species. Second, it generates gapless metabolic networks for each species based on KEGG stoichiometry data. Thus,
CoReCo allows a direct comparison between the reconstructed models, e.g., to study evolutionary aspects.

\section{MEMOSys version 2 (2014)}

MEMOSys [33] (Metabolic Model Research and development System) is a database for storing and managing genome-scale models, rather than a reconstruction tool. This tool allows tracking of changes during the development of a particular genome-scale model. Twenty genome-scale models are publicly available for exporting and modifying. Child models can be created from the 20 available models and then modified and compared with parent models. All the differences between different versions of the models can be listed to track changes in the networks.

\section{FAME (2012)}

FAME [34] (Flux Analysis and Modeling Environment) is a web-based application for creating and running GSMMs. This tool can reconstruct genome-scale models for any microorganism in the KEGG database. One of the most interesting features of FAME is that analysis results can be visualized on familiar KEGG-like maps. It is foremost a tool for running and analyzing models and is used -by us- for educational purposes. One of the limitations of FAME is that models cannot be generated for microorganisms which are not in the KEGG database.

\section{GEMSiRV (2012)}

GEMSiRV [35] (Genome-scale Metabolic Model Simulation, Reconstruction and Visualization) is a software platform for network drafting and editing. A manually curated model is used as a template to generate a draft network for the species under study. Among the tools inside the toolbox, MrBac [36] can be used to generate reciprocal orthologous-gene pairs which are then used by GEMSiRV to generate the draft model. One of the limitations of this tool is that only one template can be used per run.

\section{MetExplore (2018)}

MetExplore [37] is a web-based application for sharing and curating in a collaborative way previously reconstructed draft metabolic networks. FBA, FVA, gene, and reaction essentiality analyses can also be performed in the same environment to compare predictions with experimental data. One of the main features of this software is that networks can be automatically visualized using the lightest paths algorithm which reduces the complexity of the network.

\section{rBioNet (2011)}

This tool [38] allows assembling a metabolic reconstruction. Rather than automatically generating a draft 
metabolic network from its genome, it allows the user to either create a reconstruction from scratch or load an existing one for curation. In both cases, reference databases are used to facilitate the import of metabolite and reactions into the network. Several tests, such as deadend metabolite detection and mass and charge reaction balances, can be run to ensure high quality of the reconstruction. Finally, this tool is part of the COBRA toolbox and a tutorial of use is available for beginners.

\section{Other tools}

Microbes Flux (2012) [39], Subliminal (2011) [40], and GEMSystem (2006) [41] are no longer maintained, as confirmed by the authors of the corresponding articles.

\section{Results}

To assess the reconstruction tools, we performed both a qualitative and quantitative evaluation. As a first step, we created a list of relevant features for genome-scale reconstruction and software quality and we scored each tool depending on the performance (1: poor, 5 : outstanding). These features are related to software performance, ease of use, similarity of output networks to high-quality manually curated models and adherence to common data standards. In addition, we evaluated 18 specific features related mostly with the second stage (refinement) of the protocol for generating high-quality genome-scale metabolic reconstructions [5]. The criteria to assign a particular score in each feature is specified in Additional file 1: Table S2. Note that not all the tools were designed for the second stage, so they scored poorly on quite some features. Many of these features have not been assessed in previous reviews $[8,9]$.

Subsequently, to assess how similar the generated draft networks are to high-quality models, we reconstructed with different reconstruction tools the metabolic networks of two bacteria for which high-quality manually curated genome-scale models already were available. We chose to reconstruct the metabolic network of Lactobacillus plantarum and Bordetella pertussis, representatives of gram-positive and gram-negative bacteria, respectively. These microorganisms were selected because of three reasons. First, the corresponding GSMMs are not stored in the BIGG database, so tools that are able to use the BIGG database (AuReMe, CarveME, MetaDraft, RAVEN) in the reconstruction process cannot use the specific information for these microorganisms. If Escherichia coli or Bacillus subtilis would have been chosen instead we would have favored these tools because high-quality models for $E$. coli or B. subtilis already exist in the BIGG database and they would have been used as templates or inputs. Second, we chose these microorganisms because we were fully informed of the quality of the reconstructions as we built them ourselves and they have proven to be able to accurately replicate experimental data [11, 12, 42, 43], even by independent researchers [44, 45]. Third, these networks were reconstructed almost entirely in a manual way, so we do not expect any bias for any particular tool.

In addition to the two previous species, we also reconstructed with all the tools draft networks for Pseudomonas putida, for which four lab-independent genomescale models have been reconstructed. We compared the draft reconstructions with iJP962 [46], a model that is not in the BiGG database, that has been proven to accurately replicate experimental data and to be absent of inconsistencies [47].

The networks were generated using seven tools: AuReMe, CarveMe, Merlin, MetaDraft, ModelSEED, Pathway Tools and RAVEN. These cover most of the freely available software platforms. The general features of these tools are listed in Table 1.

\section{General assessment overview}

None of the tools got a perfect score for all of the evaluated features and usually, strengths in some tools are weaknesses in others (Fig. 1, Additional file 1: Figure S3, Tables S25 and S26 to see detailed evaluation). For example, on the one hand, ModelSEED and CarveMe were evaluated as outstanding when we checked whether the whole reconstruction process is automatic; Merlin was evaluated as poor because users should interfere more to get a network ready to perform FBA. On the other hand, we consider Merlin as outstanding with respect to a workspace for manual refinement and information to assist users during this step; CarveMe and ModelSEED do not provide further information for manual refinement nor a workspace for manual curation, so they were evaluated as poor in this category.

In some cases, all the tools got the maximum score possible. For instance, all the tested tools are properly supported by specialist teams and also maintain upto-date databases. In other cases, none of the tools got the maximum score. This was the case for automatic refinement of networks using experimental data. Some of the tools, such as ModelSEED and CarveMe, can use media composition to gap-fill the network. AuReMe and Pathway Tools also can use, in addition to media composition, known metabolic products to gap-fill the network. In spite of that, none of the tools can also use Biolog phenotype arrays, knockout experiments and different types of omics data (transcriptomic, proteomic, metabolomic, etc.) to automatically curate the network. Although some efforts have been done in this area [48-51], this seems like a major challenge for future tool development that should lead to improved metabolic reconstructions. 
Table 1 List of selected genome-scale metabolic reconstruction tools and their main features

\begin{tabular}{|c|c|c|c|c|c|}
\hline $\begin{array}{l}\text { Reconstruction } \\
\text { tool }\end{array}$ & Mapping method & $\begin{array}{l}\text { Reactions are inherited } \\
\text { from }\end{array}$ & $\begin{array}{l}\text { Associated } \\
\text { databases }\end{array}$ & Version & Type of software \\
\hline AuReMe & Pantograph (Inparanoid and OrthoMCL) & Template model(s) & BIGG-MetaCyc & 1.2 .4 & Command line \\
\hline CarveMe & Diamond, eggNOG-mapper ${ }^{a}$ & Template model & BIGG & 1.2 .1 & Command line \\
\hline Merlin & Mapping from annotation with BLAST or HMMER & Database & KEGG & 3.8 & $\begin{array}{l}\text { Standalone } \\
\text { interface }\end{array}$ \\
\hline MetaDraft & Autograph (Inparanoid) & Template model(s) & BIGG & 0.9 .2 & $\begin{array}{l}\text { Standalone } \\
\text { interface }\end{array}$ \\
\hline ModelSEED & Annotation ontology map from RAST data & Template model & ModelSEED & $2.2-2.4$ & Online service \\
\hline Pathway Tools & Pathologic & Database & MetaCyc & 22.0 & $\begin{array}{l}\text { Standalone } \\
\text { interface }\end{array}$ \\
\hline RAVEN & $\begin{array}{l}\text { Autograph-type method from BLASTP and Bidirectional } \\
\text { BLASTPB }\end{array}$ & $\begin{array}{l}\text { Database- Template } \\
\text { model(s) }\end{array}$ & KEGG-MetaCyc & 2.0 .1 & Command line \\
\hline
\end{tabular}

aeggNOG-mapper should be run externally by the user

${ }^{\mathrm{b}}$ Bidireactional BLASTP is used with template models and BLASTP with databases (KEGG and Metacyc)

Compliance with the latest SBML standards has been pointed as one of the critical points to share and represent models [52]. Consequently, we evaluated if the tools use the latest SBML features in the import (inputs) and export (outputs) of networks. For inputs, we checked if the tools were able to read networks in SBML level 3 [22]. We additionally checked if the output networks satisfy the following three features: use of SBML level 3 [22] with FBC annotations [23], SBML groups [24], and MIRIAM compliant CV annotations [22, 53]. These features are used, for example, for models in the BIGG database and they ensure that the information is stored in a standard way. For inputs, we found that among the tools that are able to import and use networks (AuReMe, MetaDraft, RAVEN) all of them are able to use SBML level 3 but AuReMe generated slightly different networks when using SBML level 2. For outputs, MetaDraft and Merlin and RAVEN were the only ones that exported the networks with all the three features. Be aware that networks created with RAVEN have to be exported to SBML using the specific functions of RAVEN (not COBRA functions as a regular COBRA user would expect) because otherwise there will be no MIRIAM annotations in the SBML files. In addition, AuReMe and CarveMe lack MIRIAM compliant CV annotations and SBML Groups, and Pathway Tools and ModelSEED exported the networks in SBML level 2.

\section{Network comparison}

We reconstructed draft networks for Lactobacillus plantarum WCFS1, Bordetella pertussis Tohama I and Pseudomonas putida KT2440 with each reconstruction tool. L. plantarum is a lactic acid bacterium (LAB), used in the food fermentation industry and as a probiotic [54-56]. Its GSMM comprises 771 unique reactions, 662 metabolites, and 728 genes, and it has been used to design a defined media for this LAB [43], to explore interactions with other bacteria [57] and as a reference for reconstructing other $\mathrm{LAB}$ [58]. In contrast to this $\mathrm{LAB}, B$. pertussis is a gram-negative bacterium, and the causative agent of the Whooping cough, a highly contagious respiratory disease [59]. The metabolic network of this pathogen was recently reconstructed, and it comprises 1672 unique reactions, 1255 metabolites, and 770 genes. As B. pertussis, Pseudomonas putida is also a gram-negative bacterium but the interest in this species relies on its capability as a cell factory to produce a wide variety of bulk and fine chemicals of industrial importance [60]. Its metabolic network comprises 1069 unique reactions, 987 metabolites, and 962 genes. While $L$. plantarum and $B$. pertussis are the main subject in the network comparisons, $P$. putida was used, as a model developed independently from us, to validate the tendencies obtained with the two previous species.

In total, 29 networks were created for L. plantarum, 27 for B. pertussis, and 27 for P. putida. The specific inputs and parameters for creating each network can be found in Additional file 1: File S1. Genes, metabolites, and reactions were extracted from the SBML files and compared with those in the manually curated model. For convenience, the manually curated model of L. plantarum, B. pertussis, and P. putida will be called hereafter iLP728, iBP1870, and iJP962, respectively.

\section{Comparison of gene sets}

Genes are the basis from which the genome-scale model is reconstructed. When a gene is included in a metabolic reconstruction, there is at least one biochemical reaction associated with that gene. When a gene is not in the reconstruction, either the reconstruction tool could not find an orthologous gene in the reference database or an orthologous gene was found but no biochemical reaction is associated with that gene. Gene sets are interesting to compare because if a gene present in the manually 


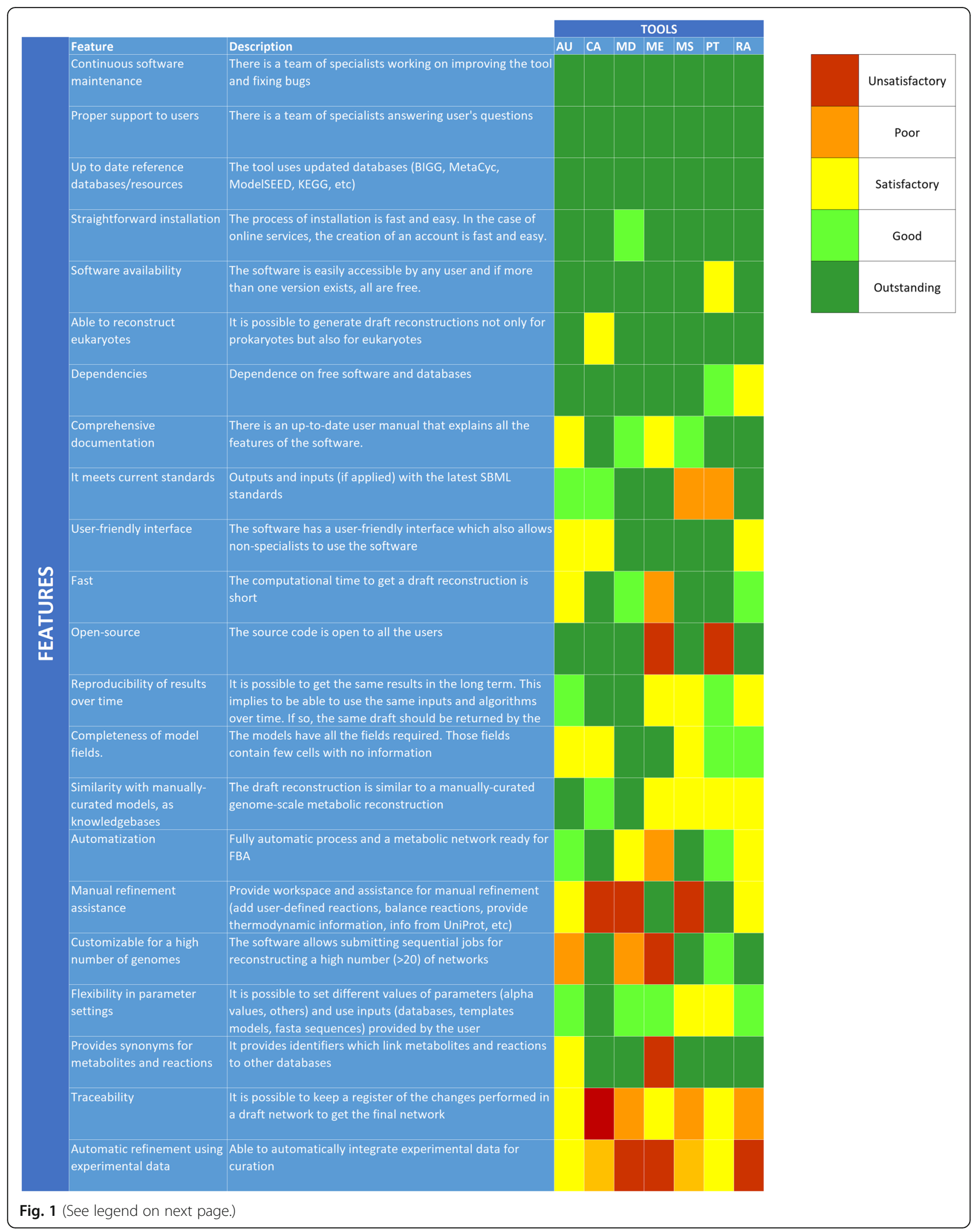


(See figure on previous page.)

Fig. 1 Qualitative assessment of the studied genome-scale metabolic reconstruction tools. We evaluated each of the tools (AU: AuReMe. CA: CarveMe. MD: MetaDraft. ME: Merlin. MS: ModelSEED. PT: Pathway Tools. RA: RAVEN) from an unsatisfactory (red) to an outstanding performance (dark green). In some categories such as continuous software maintenance and proper support, on the top of the figure, all the tools got the maximum score while in others such as automatic refinement using experimental data, none of the tools got the maximum. In most of the cases, strengths in some tools are weaknesses in others

curated model is absent in a draft reconstruction, that could explain why some biochemical reactions are missing in the draft. Alternatively, if a gene is absent in the manually curated model but present in a draft reconstruction, that could explain the presence of reactions that should not be in the reconstruction. Moreover, gene sets are simple to compare among reconstructions because gene identifiers in all the cases are the same (the locus tag in the genome annotation) and so, in contrast to metabolites and reactions, there is no mapping-related bias in the comparison.

To assess how similar the draft networks were to the corresponding manually curated networks we calculated the Jaccard distance $(J D)$ as well as the ratio between the percentage of covered genes and the percentage of additional genes $(R)$ (Additional file 1: Tables S4-S7). The $J D$ has been used before to measure the distance between genome-scale metabolic reconstructions, based on reaction sets [61]; here, we also applied it to compare reconstructions in terms of genes and metabolites. We called $J D_{g}, J D_{n}$ and $J D_{m}$ to the $J D$ between two reconstructions when they are compared in terms of genes, reactions and metabolites, respectively. Analogously, we called $R_{g}, R_{n}$ and $R_{m}$ to the $R$ when reconstructions are compared in terms of genes, reactions and metabolites, respectively. In general terms, a value of 0 in the $J D$ means that the networks are identical and a value of 1 means that the networks do not share any element. For the $R$, higher values reflect a higher similarity to the original network and lower values reflect a lower similarity with the original network.

The values in the $J D_{g}$ ranged from 0.38 to 0.60 in $L$. plantarum and from 0.43 to 0.67 in B. pertussis (Additional file 1: Tables S4 and S5), while values in the $R_{g}$ ranged from 1.18 to 13.16 in L. plantarum and from 0.84 to 3.52 in B. pertussis (Additional file 1: Tables S6 and S7). Although the similarity of the generated draft networks seems slightly better for $L$. plantarum than for $B$. pertussis, we found that it depends on which metric is analyzed. With the exception of one network, the $R_{g}$ showed that all the draft networks of $L$. plantarum were more similar to iLP728 than the draft networks of $B$. pertussis to $\mathrm{iBP} 1870$, using the analog parameter settings. In contrast, the $J D_{g}$ showed that AuReMe, ModelSEED, RAVEN, and Merlin generated draft networks of L. plantarum which are more similar to iLP728 than the draft networks of B. pertussis with regard to iBP1870, and that CarveMe, MetaDraft, and Pathway Tools generated draft networks slightly more similar for $B$. pertussis. In general, similar values of $J D_{g}$ and $R_{g}$ were obtained for P. putida (Additional file 1: File S3).

Additionally, when sorting the values of both metrics, we noticed that the $J D_{g}$ order does not correspond to that made with the $R_{g}$. The lowest $J D_{g}$ among the draft reconstructions for $L$. plantarum was obtained in the network generated with AuReMe when the gram-positive set of templates was used; for $B$. pertussis, it was obtained with MetaDraft. In contrast, the highest $R_{g}$ among the draft reconstructions for $L$. plantarum was obtained in the network generated with AuReMe when only Lactococcus lactis was used as template; for B. pertussis, it was obtained with MetaDraft when Escherichia coli template was used.

Although the similarity scores for both metrics are not entirely consistent, some trends were observed. The networks more similar, in terms of genes, to the manually curated models were generated by MetaDraft, AuReMe, and RAVEN (Fig. 2). However, since parameters settings and inputs have a big effect on the similarity scores, the usage of these tools does not automatically ensure obtaining a draft network similar, in terms of genes, to a manually curated model. This is particularly true for RAVEN which also generated some networks with high $J D_{g}$ and low $R_{g}$ scores. The same trends were obtained for P. putida (Additional file 1: Figure S2).

We further analyzed the percentage of genes covered in the manually curated models and the percentage of genes not in the manually curated models to explain differences in $R_{g}$. For all the species we observed a wide variation in both variables (Figs. 3, 4 and Additional file 1: Figure S7). Among the five networks of $L$. plantarum with the highest coverage, two were created with AuReMe and three with RAVEN; for B. pertussis, four were created with RAVEN and one with CarveMe. However, the networks created with RAVEN that recovered the highest percentages of genes also added a large number of genes which were not present in the manually curated models, decreasing the values in the $R_{g}$. In addition, AuReMe and MetaDraft created conservative draft networks with the lowest number of additional genes, which explains the higher values in the $R_{g}$. Finally, tools such as ModelSEED, Pathway Tools, and Merlin consistently created reconstructions with gene coverages not ranging in the highest values (in comparison with other 

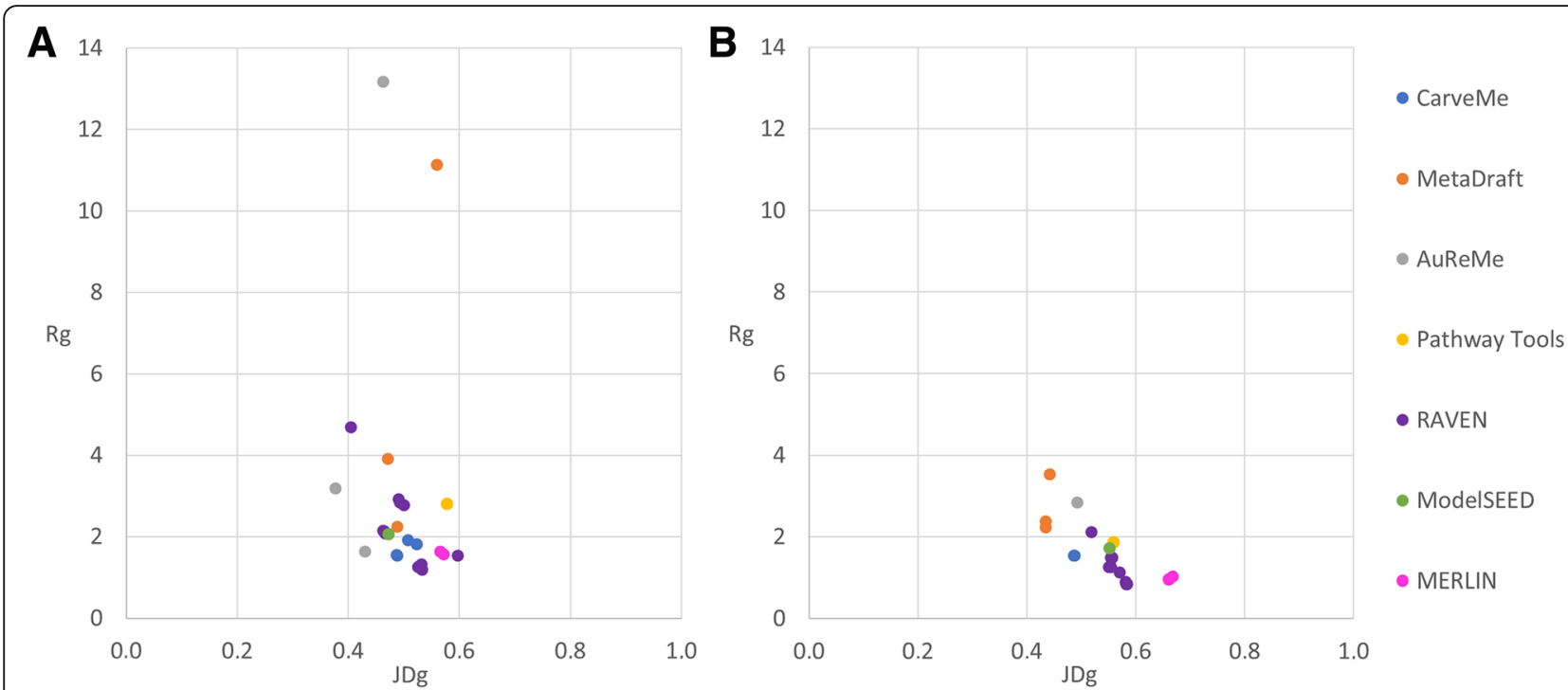

Fig. 2 Jaccard distance versus the ratio between coverage and additional genes for draft reconstructions. We used the Jaccard distance and the ratio to measure the similarity between the draft reconstructions and the corresponding manually curated models, in this case, when the networks are analyzed in terms of genes. Draft reconstructions for Lactobacillus plantarum and Bordetella pertussis are represented in panels a and b, respectively. For both cases, the networks more similar to the manually curated models are located on the top left side of each plot. Thus, the draft reconstructions more similar to the manually curated models were created by AuReMe, MetaDraft, and RAVEN

networks) and adding a relatively large number of genes not present in the manually curated models, which explains why they had lower values in the $R_{g}$.

For L. plantarum we found 1613 different genes in total with all the tools, of which 885 were not present in iLP728. For B. pertussis, 1888 different genes were found, of which 1118 were not present in iBP1870. In addition, 79 genes were correctly predicted in all the draft networks for iLP728; for iBP1870, this was 131 genes. The distribution of metabolic pathways associated to those genes is wide for both species, with carbohydrate metabolism and amino acid metabolism accounting for more than 50\% of the metabolic processes (Additional file 1: Tables S8 and S9). Additionally, 35 and 39 genes were not recovered in any network for iLP728 and iBP1870, respectively. The metabolic functions associated to those genes were very specific, with polysaccharide biosynthesis (63\%) and transport (22\%) top in the list for L. plantarum and with transport (41\%) and ferredoxin/thioredoxin related reactions (30\%) for $B$. pertussis. Finally, one gene in L. plantarum, which was associated with riboflavin biosynthesis, was recovered by all the networks but it was not present in iLP729. For $B$. pertussis, three such genes were found. These genes were associated with alternate carbon metabolism and cell envelope biosynthesis.

\section{Comparison of reaction sets}

Genes and biochemical reactions are connected within a reconstruction through gene-protein-reaction (GPR) associations. However, genes and reactions relationships are ultimately represented in reconstructions as boolean rules known as gene-reaction rules. With the exception of exchange, sink, demand, spontaneous and some transport reactions (e.g., those governed by diffusion), each reaction has a defined gene-reaction rule in the reference database used by each reconstruction tool. During the process of reconstruction, if orthologous genes are found that satisfy the gene-reaction rule of a particular reaction, that reaction is included in the draft reconstruction. Other reactions may be added to the draft reconstruction based on others criteria, such the probability of a particular pathway to exist in the microorganism under study or the need to fill particular gaps in the network in order to produce biomass. Nonetheless, we expect that networks which are more similar in terms of genes will also be more similar in terms of reactions.

In contrast to genes, however, reactions are labeled with different identifiers in different databases. Thus, the same reaction can be stored with two different identifiers in two different databases. During the reconstruction process, reactions are added from the reference database to the draft reconstruction and tools using different databases will generate reconstructions comprising reactions with different identifiers. We, therefore, used MetaNetX [62] to map reactions among reconstructions built with different databases. In this approach, reactions were compared using their identifiers (case sensitive string comparison). In addition, we compared networks using reaction equations, i.e., we 


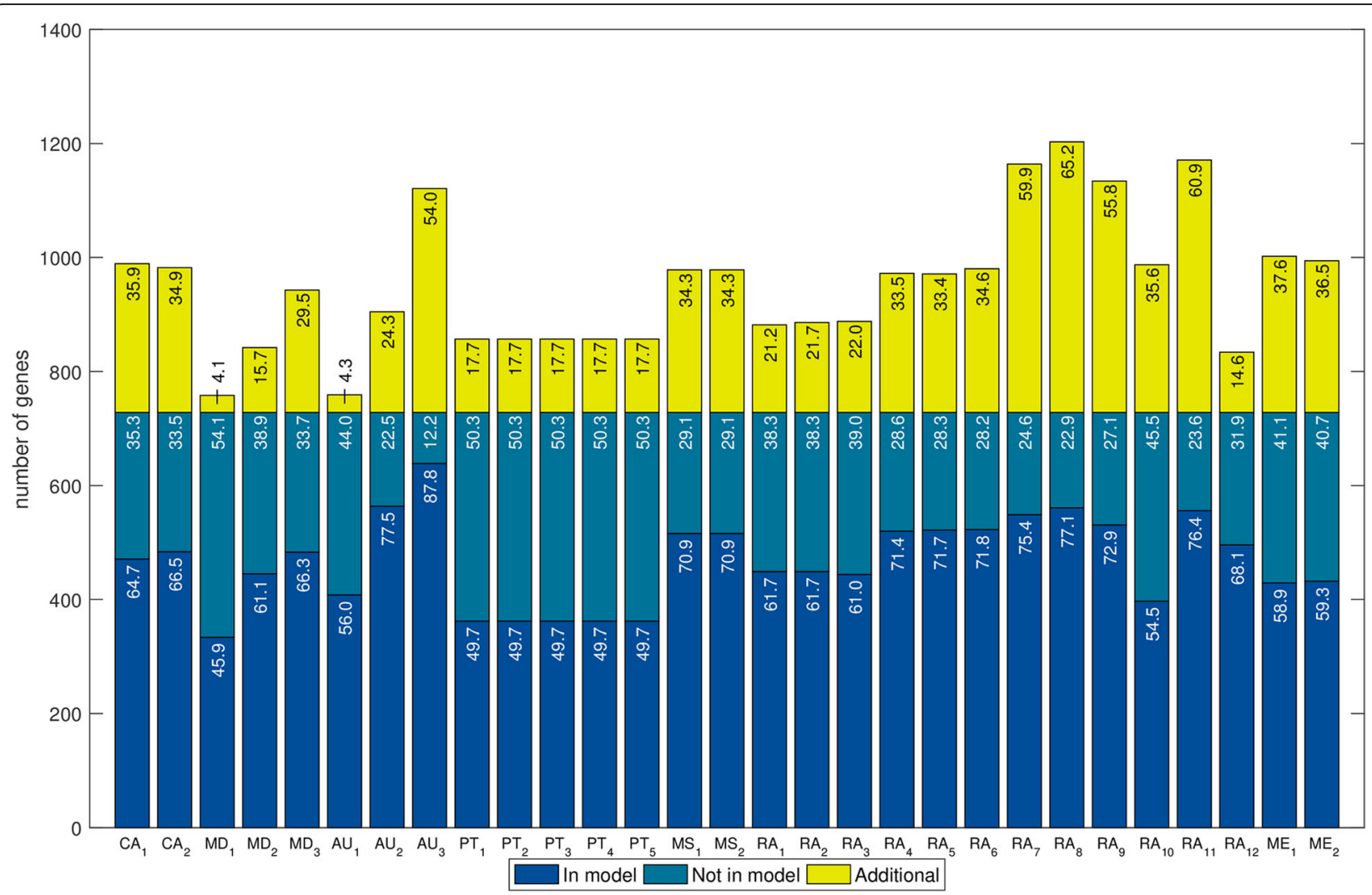

Fig. 3 Overlap of genes in draft reconstructions for Lactobacillus plantarum with those in the manually curated model. In total, 29 networks were reconstructed with 7 tools (CarveMe: CA; MetaDraft: MD; AuReMe: AU; Pathway Tools: PT; ModelSEED: MS; RAVEN: RA; Merlin: ME). Several reconstructions, which are represented with different sub-indices, were generated for each tool using different parameters settings. Numbers inside bars represent percentages with respect to the total number of genes in iLP728. The coverage (blue bars) ranged from 49.7 to $87.8 \%$ while the percentage of additional genes (yellow bars) ranged from 4.3 to $65.0 \%$. Most of the genes that were not recovered (dark green bars) are related to very specific metabolic functions that were carefully incorporated during the manual curation of iLP728 such as polysaccharide biosynthesis and transport

compared reactions using their attributes instead of their identifiers. In this second approach, we considered that two reactions were the same if they had the same metabolites with the same stoichiometric coefficients. Some exceptions were made to also match reactions that differ only in proton stoichiometry (due to differences in metabolites charge) or to catch reactions which are written in the opposite direction (reactants in the side of products). We decided to include exchange reactions in the network comparison for completeness because CarveMe and ModelSEED automatically generate them; as they are non-gene associated reactions, this automatically lowers the scores for the other tools that do not add exchange reactions. For most networks, comparison through reaction identifiers resulted in a lower percentage of coverage than through reaction equation comparison (Additional file 1: Tables S10 and S11). This lower coverage was due to some missing relationships between different databases in MetaNetX, which we discovered when comparing with the reaction equations. In total,
220 new unique reaction synonyms pairs were automatically discovered for both species with the second approach (Additional file 1: Table S12). To further overcome the missing relationships in MetaNetX, a semi-automatic algorithm was developed to assist the discovery of new metabolite synonyms. In total, 187 new metabolites synonyms were discovered (Additional file 1: Table S13) which led to the discovery of 282 additional reaction synonyms (Additional file 1: Table S14).

The comparison through reaction equations showed a wide variation in reaction coverage and percentage of additional reactions for all the species (Figs. 5 and 6 and Additional file 1: Figure S8). In addition, for those networks created with RAVEN (KEGG), ModelSEED, and Merlin, we observed a considerable number of reactions with a partial match with the manually curated model. These partial matches emerge from differences in proton stoichiometry, which indicates the existence of metabolites with different charge than those found in the manually curated models. In contrast to the gene sets comparison, where the coverage 


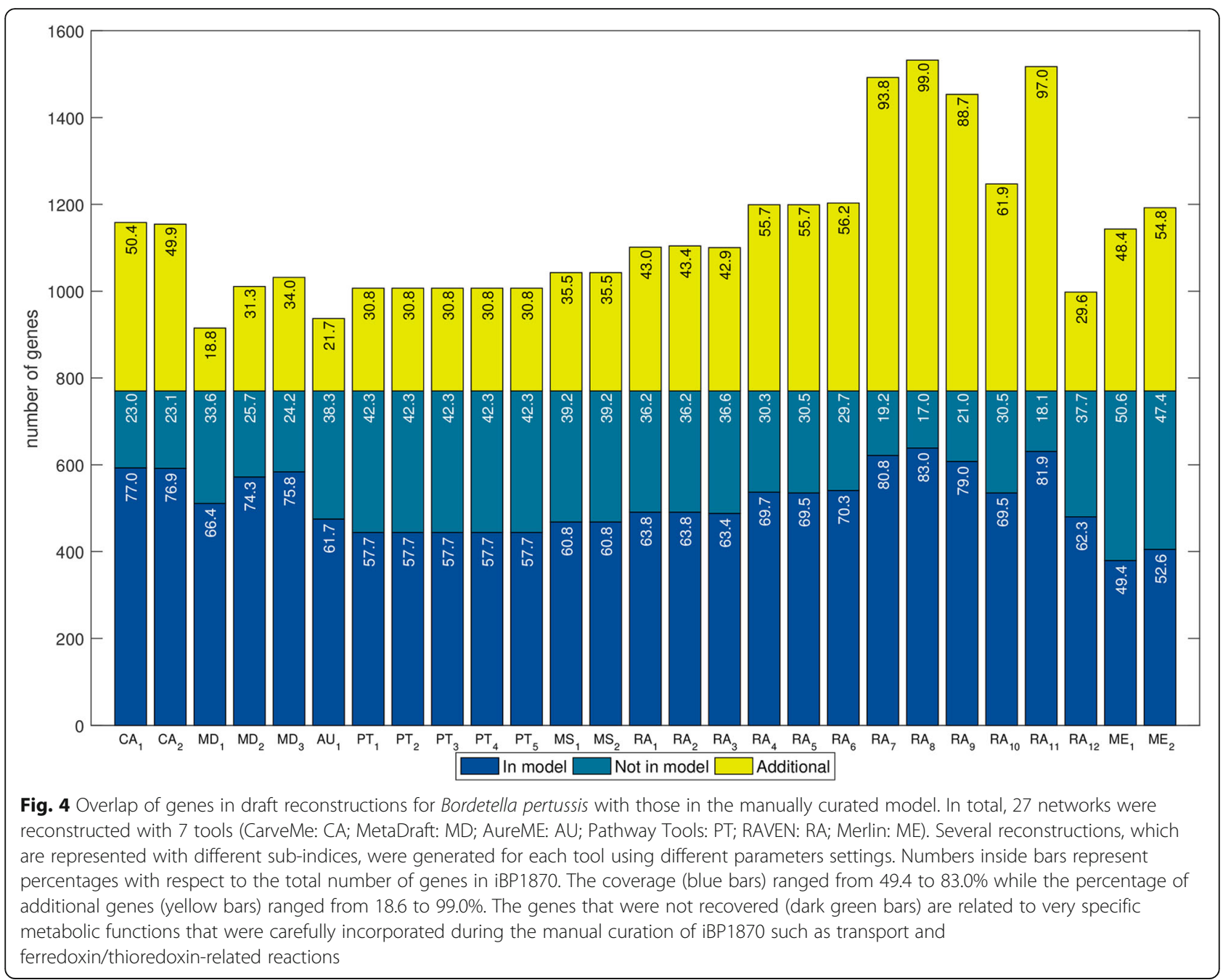

was as high as $88 \%$ and $83 \%$, we only observed a maximum coverage of $72 \%$ and $58 \%$, for L. plantarum and B. pertussis, respectively, even when considering partial matches. We classified the reactions that were not recovered in different categories (Additional file 1: Figures S3-S6) and we found that the low reaction coverage can be explained mainly by three reasons.

First, both manually curated models contain a considerable amount of reactions without gene-associations, including spontaneous, transport, exchange reactions, reactions added during the manual gap-filling and biomass-related reactions. For L. plantarum and B. pertussis, there are 241 and 657 of such reactions, representing $31 \%$ and $39 \%$ of the network, respectively. With the exception of CarveMe and ModelSEED, which can perform automatic gap-filling, all the rest of the tools are not able to recover most of the non-gene associated reactions, mainly because all the tools predict reactions based on genomic evidence. Thus, for both species, around, $50 \%$ of the reactions that were not recovered do not have gene-reaction associations in the manually curated model. Without considering exchange reactions, the coverage roughly increased by $15 \%$ and $12 \%$ for $L$. plantarum and B. pertussis, respectively, except for CarveMe and ModelSEED. Second, in around 30\% of the reactions that were not recovered, there are at least $50 \%$ of the associated genes missing in the draft reconstructions. Third, even when all the genes associated with a particular reaction are recovered, specific substrate and cofactor usage is difficult to predict. Many times, the tools predict the correct metabolic activity but they fail in predicting the specific substrate used in the manually curated models. We created a collection of plain text files containing hundreds of examples where the associated genes were recovered by the tool but the reaction does not correspond to the one in the manually curated model because of different substrates (see section availability of data for details).

We again calculated the $J D_{r}$ and the $R_{r}$ to assess how similar the networks were, in this case in terms of 


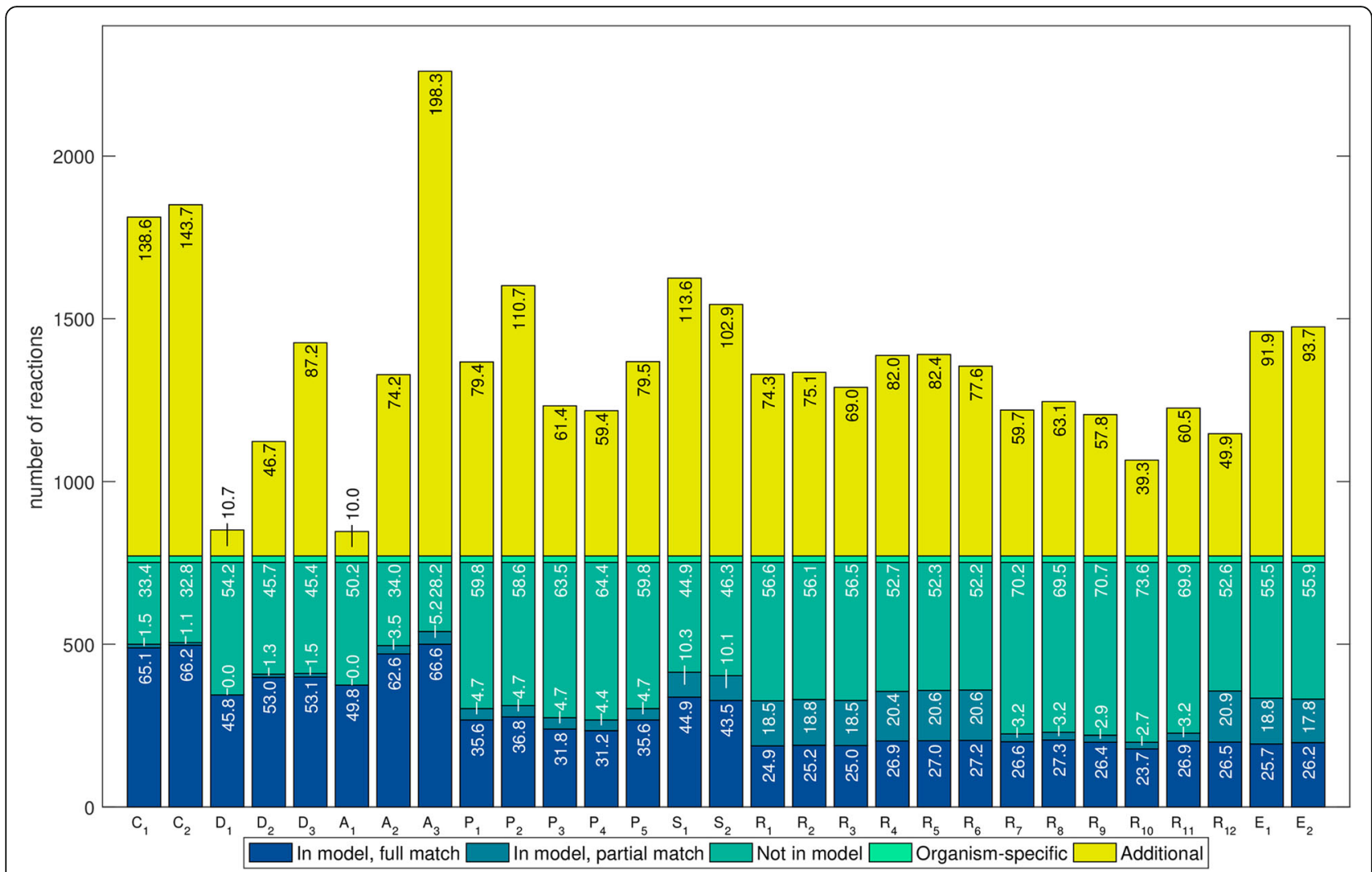

Fig. 5 Overlap of reactions in draft reconstructions for Lactobacillus plantarum with those in the manually curated model. In total, 29 networks were reconstructed with 7 tools (CarveMe: C, MetaDraft: D, AuReMe: A, Pathway Tools: P, ModelSEED: S, RAVEN: R, Merlin: E). Several reconstructions, which are represented with different sub-indices, were generated for each tool using different parameters settings. Numbers inside bars represent percentages with respect to the corrected number of reactions in iLP728, which is the total number of reactions in iLP728 minus the biomass-related reactions (light green). We observed a wide variation in the coverage (blue bars) and the percentage of additional reactions (yellow bars). In addition, a considerable number of reactions in the networks build with ModelSEED, RAVEN (KEGG), and Merlin contained different stoichiometry for protons than those in iLP728 (dark green bars)

reactions. The first observation we made is that, independent of the metric and for both species, each reconstruction was less similar in terms of reactions than in terms of genes, which is consistent with the decrease in coverage. In addition, as in the gene comparison, the order of scores for the $R_{g}$ and the $R_{r}$ by magnitude was not the same. If we compare the similarity scores for reaction sets with the ones for gene sets, we see almost the same trend but with one difference. AuReMe and MetaDraft are still the tools with the best similarity scores but now CarveMe goes up in the list of scores and RAVEN goes down (Fig. 7, Additional file 1: Tables S4-S7). This was particularly true for B. pertussis where two networks reconstructed with CarveMe got the two first places in the $J D_{r}$ list. Almost the same trend was observed for P. putida (Additional file 1: Figure S2) being the higher scores for RAVEN instead of CarveMe the main difference.

Although RAVEN generated some reconstructions with high gene sets similarity to the manually curated models, it did not for reaction sets similarity. We, therefore, analyzed one of the networks reconstructed with RAVEN in more detail, one that was consistently in the top 5 list for both species for both metrics. We found one main reason for the decrease in performance. The analyzed network was created based on KEGG, so metabolites were not labeled as intracellular or extracellular. Hence, no transport or exchange reactions were present. Although there are functions to incorporate this kind of reactions in RAVEN, that is considered as manual curation because users must specify which compounds should be transported, and we here only tested how much work would it take to transform these draft networks into high-quality reconstructions.

We further analyzed reactions that were present and absent in all the reconstructions to understand which kind of metabolic processes they were related. Sixty-six reactions in iLP728 and 98 in iBP1870 were always found in all the draft networks. In agreement with the gene sets analysis, the associated metabolic processes are mainly amino acid metabolism, nucleotide metabolism, and carbohydrate metabolism (Additional file 1: Tables 


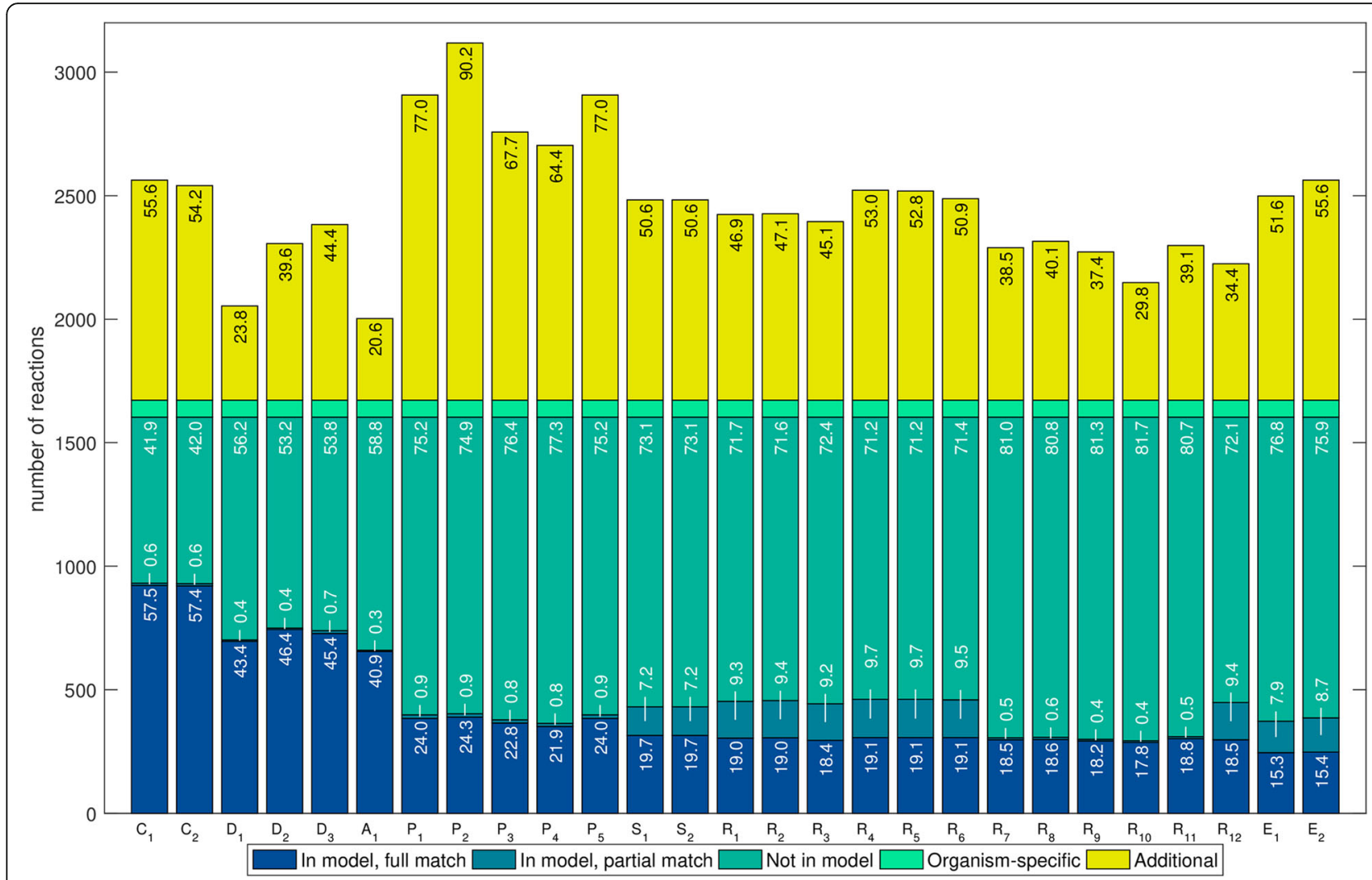

Fig. 6 Overlap of reactions in draft reconstructions for Bordetella pertussis with those in the manually curated model. In total, 27 networks were reconstructed with 7 tools (CarveMe: C, MetaDraft: D, AuReMe: A, Pathway Tools: P, ModelSEED: S, RAVEN: R, Merlin: E). Several reconstructions, which are represented with different sub-indices, were generated for each tool using different parameters settings. Numbers inside bars represent percentages with respect to the corrected number of reactions in iBP1870, which is the total number of reactions minus the biomass-related reactions (light green). We observed a wide variation in the coverage (blue bars) and the percentage of additional reactions (yellow bars). In addition, a considerable number of reactions in the networks build with MODELSEED, RAVEN (KEGG), and Merlin contained different stoichiometry for protons than those in iBP1870 (draft green bars)

S15 and S16). Additionally, 165 reactions in iLP1870 and 598 in iBP1870 were not found by any tool. In both species, around $10 \%$ of those reactions were biomass-related reactions and from the rest, most of them were exchange reactions, transport reactions without gene associations and reactions in other categories that were not in the BIGG database (Additional file 1: Tables S17 and S18). Only one reaction, associated to amino acid metabolism, was found in all the draft networks of $L$. plantarum but not in iLP728; four reactions, associated mainly to carbohydrate metabolism, were found in all the draft networks but not in iBP1870.

\section{Comparison of metabolite sets}

Other important elements within metabolic reconstructions are metabolites. When a biochemical reaction is added to the draft network during the reconstruction process, all the reactants and products are added to the network too. As the draft metabolic networks were created with different tools, each of which using its own set of databases, they had different identifiers for the same metabolite. For those networks whose identifiers were different from BIGG, we again used MetaNetX and our own additional dictionary to map metabolites.

We calculated the $J D_{m}$ and the $R_{m}$ to assess the metabolite sets similarity. For almost all the draft networks in both species, the values in the $J D_{m}$ were between the $J D_{g}$ and the $J D_{r}$; we found the same for the $R_{m}$ (Additional file 1: Tables S4-S7). Again, when sorting the networks according to their metric scores, we found the same trends than for reaction sets. The first position in the lists were networks either reconstructed with MetaDraft, AureMe, or CarveMe. Moreover, independently of the metric and the species, MetaDraft reconstructed $40 \%$ of the networks among those in the top 5 .

Two hundred six metabolites in iLP728 and 271 in iBP1870 were correctly predicted in all the draft networks. These metabolites were in both cases mainly associated with carbohydrate metabolism and amino acid metabolism (Additional file 1: Tables S19 and S20). Eighty-one metabolites in iLP728 and 278 in iBP1870 were not recovered in any network. Of those, 16 were 

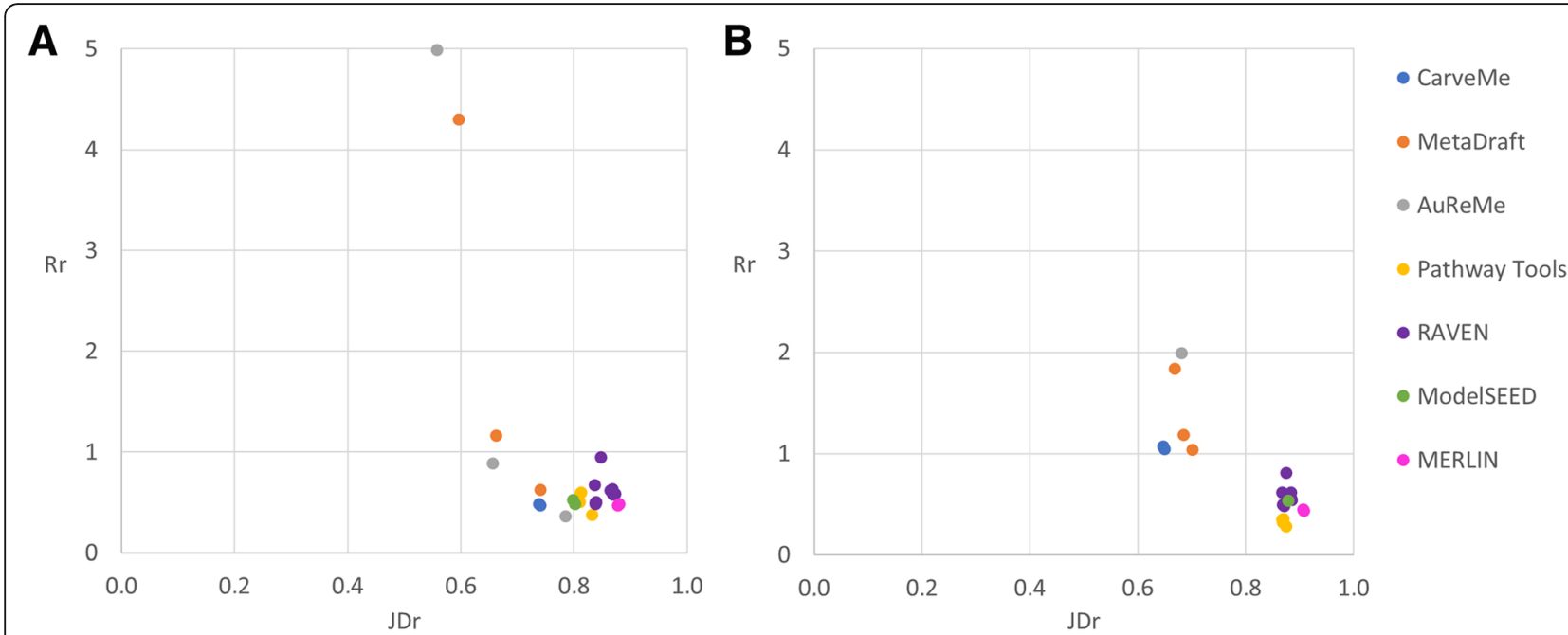

Fig. 7 Jaccard distance versus the ratio between coverage and percentage of additional reactions for draft reconstructions. We used the Jaccard distance and the ratio to measure the similarity between the draft reconstructions and the corresponding manually curated model, in this case, when the networks are analyzed in terms of reactions. Draft reconstructions for Lactobacillus plantarum and Bordetella pertussis are represented in panels $\mathbf{a}$ and $\mathbf{b}$, respectively. For both cases, the networks more similar to the manually curated models are located on the top left side of the plot. Thus, the draft reconstructions more similar, in terms of reactions, to the manually curated models were created by AuReMe, MetaDraft, and CarveMe

related to the biomass of $L$. plantarum and 16 others were not in the BIGG database. For iBP1870, 44 were biomass-related and 47 others were not in the BIGG database. Finally, 9 and 11 metabolites were recovered in all the networks but they were not present in iLP728 and iBP1870, respectively. Mainly, they were associated to the metabolism of cofactors and vitamins and amino acid metabolism in the case of $L$. plantarum and carbohydrate metabolism and glycan biosynthesis in the case of B. pertussis (Additional file 1: Tables S21 and S22).

\section{Topological analysis}

To compare the topological features of each network, we calculated the number of dead-end metabolites, the number of orphan reactions, the number of unconnected reactions and other metrics (Additional file 1: Tables S23 and S24).

iLP728 has 113 dead-end metabolites while iBP1870 has 59 . This is consistent with the observation that many pathways are disrupted in L. plantarum leading for example to well-known auxotrophies for many amino acids $[42,43]$. With the exception of CarveMe, all the tools generated networks with a high number of dead-end metabolites, ranging from 244 and 999 , and from 379 to 976, for L. plantarum and B. pertussis, respectively. The low number of dead-end metabolites in CarveMe is caused by the use of a manually curated universal model as a template which lacks dead-end metabolites.

Without considering exchange and demand/sink reactions, 127 and 449 reactions without gene associations (called orphan reactions) were found in iLP728 and iBP1870, respectively. These reactions are mainly associated with transport amino acid metabolism, and biomass formation. MetaDraft, AuReMe, and RAVEN returned metabolic networks with no orphan reactions. These tools only include reactions with genomic evidence and others lacking this support are not included. ModelSEED returned networks with a low amount of orphan reactions, which are related to exchange reactions. In contrast, CarveMe, Pathway Tools, and Merlin returned networks with a significantly larger number of orphan reactions (ranging from 66 to 491 in L. plantarum and from 115 to 736 in $B$. pertussis). For CarveMe, this is due to the inclusion of transport and spontaneous reactions as well as reactions needed to create biomass (from gap-filling); for Pathway tools, it is because of the addition of reactions to complete probable pathways and spontaneous reactions; and for Merlin, this is solely due to spontaneous reactions.

\section{Discussion}

In this work, we reviewed the current state of all the reconstruction tools we could find in the literature and performed a systematic evaluation of seven of them. None of the tools performed well in all the evaluated categories so users should carefully select the tool(s) that suit the purpose of their investigation. For example, if a high-quality draft is required and models are available for a phylogenetically close species, MetaDraft or AuReMe could be selected, reducing thus the time needed to obtain a high-quality manually curated model. Of these, MetaDraft was the most robust for handling models and 
since it has a graphical user interface, it is also suitable for non-specialists. AuReMe, on the contrary, offered a command-line workspace where the traceability is the priority. Although we were not able to use RAVEN in the template mode (for details, see error messages in supplementary files), this tool allowed us to automate the generation of several reconstructions, it had a high flexibility with parameters and it offered integration with the KEGG and MetaCyc databases which makes it very appropriate for less-studied species. ModelSEED, CarveMe, and Pathway Tools were the fastest tools to generate reconstructions having a great potential for largescale studies how it has been proven in previous works $[61,63]$. The first two tools provided networks which are ready to perform FBA, however presumably because of the automatic gap-filling procedure, too many reactions that should be manually verified must be expected. Pathway Tools and Merlin provided platforms suitable for manual curation which nicely guide the user through the whole reconstruction process.

The list of features that we defined not only can be used by model builders to select the best tool(s) but also by developers as a guide for improving them. We highlight four features, which are in accordance with the FAIR guiding principles for scientific data management and stewardship [64], that should be considered as a priority by developers to ensure management of reconstructions in a standard way: (1) To be findable: all the genes, metabolites and reaction in a reconstruction should be assigned with unique and persistent identifiers, and synonyms or aliases in other databases should be provided whenever possible. (2) To be accessible: exhaustive control of versions should be implemented so users will be able to submit small but significant changes to draft reconstructions, to trace changes made during the reconstruction process, or to retrieve a particular version if desired. (3) To be interoperable: output (and input if applied) reconstructions should be written with the latest features of the SBML standards. (4) To be reusable: in relationship with providing a detailed provenance, transparency of decisions through the whole reconstruction process should be ensured so users can see why a particular reaction was added and at which stage (draft network generation, gap-filling, refinement, etc.).

Genome-scale reconstructions are usually evaluated after they are converted into genome-scale models [5], i.e., mathematical structures where simulations can be performed under constraints that describe specific experimental conditions. Thus, GSMMs are tested by their accuracy to predict experimental data such as knockouts, nutritional requirements and growth rates on different conditions. However, most of the drafts we generated were not suitable to perform FBA, mainly due to the lack of biomass-related, transport and exchange reactions. Thus, we limited the evaluation of the drafts to the comparison with manually curated, genome-scale reconstructions. The latter are valuable by themselves as knowledgebases because they contain extensive information from the literature. Here, we prescribed that the manually curated reconstructions are the gold standard, which implies that they cannot be improved and that is obviously not true. Many reconstructions of, for example, E. coli, S. cerevisiae, and H. sapiens have gone through multiple rounds of improvements during the years [65-67]. As reference databases used by reconstruction tools increase in size and quality, so will the reconstructions which are based on them. Therefore, some of the reactions which were suggested by the tools and which are not in the manually curated models could indeed be reactions which would improve the quality of the reconstructions. Whether one of those reactions should be in the reconstruction or not will depend not only on the genomic evidence but also on the scope and context of the reconstruction. Many reactions are usually not incorporated because they are not needed for modeling purposes [5]. Thus, similarity scores should not be taken alone to assess the quality of draft reconstructions. Indeed, additional reconstructions of Lactobacillus plantarum that we made with CarveMe and ModelSEED and which were gap-filled using a modified version of CDM (Additional file 1: File S2), a media that support the growth of this microorganism in vivo [43], showed a general performance close to the manually $\mathrm{cu}$ rated model, suggesting that although the networks are not so similar as others created with different tools, the core metabolism remains similar. Despite that, the performance of these networks is dependent on the media composition which is used for the gap-filling (Additional file 1: Figure S1), and therefore if there is no experimentally determined media, some false positive and false negative predictions could emerge. For example, if very accurate predictions regarding nutritional requirement are needed to design a microbial community, automatic reconstructions for which an experimentally determined media composition is not provided during gap-filling could result in false predictions.

A correct mapping of identifiers among different databases is crucial to perform a proper comparison between metabolic networks. Important efforts such as MetaNetX [62] and Borgifier [68] have been done to facilitate this titanic task. The first of those tools allowed us to map most of the metabolites and reactions among the different reconstructions but naturally, some relationships were missing. To overcome this limitation, we fully mapped metabolites in the manually curated models to known databases namely BiGG, KEGG, MetaCyc, and SEED. Second, we implemented an algorithm to search reaction equations, even when they have differences in proton stoichiometry due to different protonation states 
or even if the reactions are written in the opposite direction. As a third step to further reduce the fraction of metabolites which were not mapped and through a semi-automatic and iterative process, we determined 187 new relationships. In spite of our efforts, some relationships were still missing which evidence the complexity of the problem. Since recent efforts have made clearer the type of issues arising in different databases [69], we emphasize the importance of standards, which could make easier the identification of synonyms because of the presence of high-quality information, and the need of an outstanding mapping system.

Systematic assessments of tools for systems biology have become very popular $[70,71]$ due to the great impact they have in the community of potential users who certainly are searching the best tool to apply in their research. Knowing the strengths and limitations of each tool allow users to select the best tool(s) for their case, to save time in preliminary tests and to focus more on the analysis and modeling using those reconstructions. Moreover, to provide genome-scale models of high quality, in terms of usability and standards, has become a priority during the last years. Efforts such as those done by Memote [52] highlight the need for suites that test the quality of genome-scale models to ensure high-quality outputs, not only in terms of their content as knowledgebases but also in terms of standards.

\section{Conclusions}

All the assessed reconstruction tools showed strengths and weaknesses in different areas and none of the tools outperformed the others in all the categories. In particular, template-based reconstruction tools such as AuReMe, MetaDraft, and CarveMe generated networks with a higher reaction sets similarity to manually curated networks than other tools. In addition, tools such as Pathway Tools and Merlin provide a proper workspace and useful information for manual refinement which could be suited for cases where much time can be dedicated to this step. RAVEN provides a platform in which biochemical information from different databases and approaches can be merged, which could be useful for less characterized species. Finally, tools such as CarveMe and ModelSEED provide ready-to-use metabolic networks which can be useful for a fast generation of model-driven hypothesis and exploration but users will have to be aware of potential false results.

There seems to be a trade-off between coverage and similarity, and it remains to be seen how much room for improvement there is. We see three clear features that would improve any tool: better standards that would allow easier integration of the best of tools, exhaustive version control during the reconstruction process, and algorithms that can use experimental data for inclusion of genes and reactions into the models.

\section{Materials and methods \\ Protein sequences}

We used the protein sequences or the GenBank files of the different microorganisms as input to generate the genome-scale metabolic reconstructions with each of the selected tools. All the protein sequences were downloaded from NCBI. For Lactobacillus plantarum strain WCFS1, Bordetella Pertussis strain Tohama I, and Pseudomonas putida KT2440 we used the protein sequences deposited under the NCBI accession numbers NC_004567.2 [72, 73], NC_002929.2 [74, 75] and NC_ 002947.4 [76, 77] respectively.

\section{Reconstruction}

The specific parameters and inputs used to reconstruct the draft networks with each tool can be found in Additional file 1: File S1.

\section{AuReMe}

We used AuReMe version 1.2.4, which was downloaded using Docker Toolbox, to generate the draft reconstructions.

To generate the genome-scale metabolic reconstructions of Lactobacillus plantarum we used three different set of templates from the BIGG database: (1) Lactococcus lactis (iNF517). (2) Lactococcus lactis (iNF517), Bacillus subtilis (iYO844), Staphylococcus aureus (iSB619), Clostridium ljungdahlii (iHN637) and Mycobacterium tuberculosis (iNJ661). 3) Lactococcus lactis (iNF517), Bacillus subtilis (iYO844), Staphylococcus aureus (iSB619), Clostridium ljungdahlii (iHN637), Mycobacterium tuberculosis (iNJ661), Escherichia coli (iML1515), Klebsiella pneumoniae (iYL1228), Shigella flexneri (iSFxv_1172), Shigella boydii (iSbBs512_1146), Shigella sonnei (iSSON_1240), Pseudomonas putida (iJN746), Yersinia pestis (iPC815), Helicobacter pylori (iIT341), Geobacter metallireducens (iAF987), Salmonella entérica (STM_ v1_0), Thermotoga maritima (iLJ478), Synechocystis sp (iJN678), and Synechococcus elongatus (iJB785).

For Bordetella pertussis we used Escherichia coli as a template (iML1515).

For Pseudomonas putida we used Pseudomonas putida as a template (iJN746).

\section{CarveMe}

We used CarveMe version 1.2.1 (downloaded from https://github.com/cdanielmachado/carveme on August 1st, 2018) to generate the draft reconstructions. Two genome-scale metabolic reconstructions were generated for Lactobacillus plantarum using the universal bacterial template and the gram-positive 
bacterial template, respectively. For $B$. pertussis, the universal bacterial template and the gram-negative bacterial template were used. For $P$. putida, the universal bacterial template and the gram-negative bacterial template were used.

\section{Merlin}

We used Merlin version 3.8 (downloaded from https:// merlin-sysbio.org/index.php/Downloads on August 1st, 2018) to generate the draft reconstructions. For all the networks, we first annotated the genomes with EBI through MERLIN using default parameters. Then, we loaded KEGG metabolic data and integrated the annotation with the model. Finally, we created gene-reactionprotein associations and removed unbalanced reactions to be able to export the network to SBML format.

\section{MetaDraft}

We used MetaDraft version 0.9.2, which was obtained from https://systemsbioinformatics.github.io/cbmpymetadraft/.

To generate the genome-scale metabolic reconstructions of Lactobacillus plantarum we used three different set of templates from the BIGG database: (1) Lactococcus lactis (iNF517). (2) Lactococcus lactis (iNF517), Bacillus subtilis (iYO844), Staphylococcus aureus (iSB619), Clostridium ljungdahlii (iHN637) and Mycobacterium tuberculosis (iNJ661). (3) Lactococcus lactis (iNF517), Bacillus subtilis (iYO844), Staphylococcus aureus (iSB619), Clostridium ljungdahlii (iHN637), Mycobacterium tuberculosis (iNJ661), Escherichia coli (iML1515), Klebsiella pneumoniae (iYL1228), Shigella flexneri (iSFxv_1172), Shigella boydii (iSbBs512_1146), Shigella sonnei (iSSON_1240), Pseudomonas putida (iJN746), Yersinia pestis (iPC815), Helicobacter pylori (iIT341), Geobacter metallireducens (iAF987), Salmonella entérica (STM_ v1_0), Thermotoga maritima (iLJ478), Synechocystis sp (iJN678), and Synechococcus elongatus (iJB785).

To generate the genome-scale metabolic reconstructions of Bordetella pertussis we used three different set of templates from the BIGG database: (1) Escherichia coli (iML1515). 2) Escherichia coli (iML1515), Klebsiella pneumoniae (iYL1228), Shigella flexneri (iSFxv_1172), Shigella boydii (iSbBs512_1146), Shigella sonnei (iSSON_1240), Pseudomonas putida (iJN746), Yersinia pestis (iPC815), Helicobacter pylori (iIT341), Geobacter metallireducens (iAF987), Salmonella entérica (STM_ v1_0), Thermotoga maritima (iLJ478), Synechocystis sp (iJN678), and Synechococcus elongatus (iJB785). 3) Escherichia coli (iML1515), Klebsiella pneumoniae (iYL1228), Shigella flexneri (iSFxv_1172), Shigella boydii (iSbBs512_1146), Shigella sonnei (iSSON_1240), Pseudomonas putida (iJN746), Yersinia pestis (iPC815), Helicobacter pylori (iIT341), Geobacter metallireducens
(iAF987), Salmonella entérica (STM_v1_0), Thermotoga marítima (iLJ478), Synechocystis sp (iJN678), Synechococcus elongatus (iJB785), Lactococcus lactis (iNF517), Bacillus subtilis (iYO844), Staphylococcus aureus (iSB619), Clostridium ljungdahlii (iHN637), and Mycobacterium tuberculosis (iNJ661).

To generate the genome-scale metabolic reconstructions of Pseudomonas putida, we used three different set of templates from the BIGG database: (1) iJN746. (2) iJN746 iML1515 - iYL1228 - iSFxv_1172 - iSbBS512_1146 iSSON_1240 - iPC815 - STM_v1_0 - iIT341 - iAF987 iLJ478 - iJN678 - iJB785 iJN746 - iML1515 - iYL1228 iSFxv_1172 - iSbBS512_1146 - iSSON_1240 - iPC815 STM_v1_0 - iIT341 - iAF987 - iLJ478 - iJN678 - iJB785 iNF517 - iYO844 - iSB619 - iHN637 - iNJ66.

\section{ModelSEED}

We used ModelSEED version 2.2 web service on August 16st of 2018 to generate the draft reconstructions of Lactobacillus plantarum and B. pertussis. Version 2.4 was used to generate the draft reconstructions for Pseudomonas putida. Models were created using different template models. No media was specified to create the models.

\section{Pathway Tools}

We used Pathway Tools version 22.0 to generate the draft reconstructions. Four networks were created with the Desktop mode using different cutoff values for pathways prediction and one was made with the Lisp-console with default parameters. All the networks were exported manually with the Desktop mode.

\section{RAVEN}

We used RAVEN version 2.0.1, which was downloaded from https://github.com/SysBioChalmers/RAVEN, to generate the draft reconstructions. Different models were created using different databases (KEGG and MetaCyc) and different values in the parameters for orthology searches.

\section{Pre-processing of $L$. plantarum and $B$. pertussis network}

We pre-processed the manually curated networks in order to compare them with the draft networks. We semi-automatically changed metabolite and reaction identifiers to match those of the BIGG database. Also, we removed duplicated reactions (those with the same reaction equation). Before the deletion of a duplicated reaction, the associated gene-reaction rule was transferred to or merged with the gene-reaction rule of the reaction that was kept in the network. 


\section{Comparison of gene sets}

We define the union of all the unique genes found in a particular metabolic network as the gene set in that network. We compared gene sets from each draft network with those in the corresponding manually curated model by case sensitive string comparison.

\section{Comparison of metabolite sets}

Each metabolic network contains a set of metabolites. For those networks generated with reconstruction tools using the BIGG database (AuReMe, CarveMe, and MetaDraft), we compared metabolites just by string comparison. For other reconstruction tools (Merlin, ModelSEED, Pathway Tools, and RAVEN), we mapped the metabolites using MetaNetX version 3.0 [62]. As metabolite identifiers in the manually curated models contain at the end of the string a character describing the specific compartment in which the metabolite is located (for example glc_c for glucose in the cytoplasmic space) and in MetaNetX they do not, we used the following procedure to compare metabolites: For each metabolic network and for each metabolite we removed the compartment char from the metabolite identifier. Then, if the modified identifier is present in MetaNetX and if there is a synonym for that identifier in the BIGG database, we checked if some of the BIGG synonyms concatenated with the before removed compartment char match a metabolite in the manually curated model. If so, we considered that the metabolite is present in the manually curated model. Otherwise, we considered that the metabolite is not present.

\section{Comparison of reaction sets}

Each metabolic network contains a set of reactions. Reaction sets were compared using two complementary methodologies. First, by using reaction identifier MetaNetX mapping and second, by using reaction equation comparison.

In the first approach, as a pre-processing step, we removed duplicated reactions (those reactions with the same MetaNetX identifier even if the reaction equation is different). For those networks generated with reconstruction tools using the BIGG database (AuReMe, CarveMe, and MetaDraft) reactions identifiers were compared by direct case sensitive string comparison. For other reconstruction tools, MetaNetX was used to map reaction identifiers, which also were compared by string comparison.

In the second case, as a pre-processing step, we first removed duplicated reactions (those with the same equation even if they had different identifiers) and empty reactions (those with an identifier but with no reactants and products). Then, reaction equations were compared by comparing each metabolite and its stoichiometry individually. For those networks generated with reconstruction tools using the BIGG database (AuReMe, CarveMe, and MetaDraft), we directly compared reaction equations. For those networks generated with reconstruction tools using a database different from BIGG (Merlin, ModelSEED, Pathway Tools, and RAVEN), we first converted metabolite identifiers to BIGG by using MetaNetX version 3.0 and our own dictionary (Additional file 1: Table S13). Then, reaction equations were compared.

All the comparison was done in MATLAB and model handling was performed using functions from Cobra Toolbox v.3.0 [14].

\section{Calculation of Jaccard distance}

The Jaccard distance $(J D)$ was calculated to compare reconstructions in terms of genes, reactions and metabolites. For two any sets of elements, $S_{i}$ and $S_{j}$, the $J D$ is calculated as $J D=1-\left|S_{i} \cap S_{j}\right| /\left|S_{i} \cup S_{j}\right|$. We called $J D_{g}, J D_{r}$ and $J D_{m}$ to the $J D$ calculated in terms of genes, reactions and metabolites, respectively. Thus, $J D_{g}, J D_{n}$ and $J D_{m}$ were calculated as:

$J D_{g}=1-\left|G_{i} \cap G_{r e f}\right| /\left|G_{i} \cup G_{r e f}\right|, G_{i}$ being the genes set of the generated draft network $i$ and $G_{r e f}$ being the genes set of the reference network (manually curated model).

$J D_{r}=1-\left|R_{i} \cap R_{\text {ref }}\right| /\left|R_{i} \cup R_{r e f}\right|, R_{i}$ being the reactions set of the generated draft network $i$ and $R_{\text {ref }}$ being the reactions set of the reference network (manually curated model).

$J D_{m}=1-\left|M_{i} \cap M_{\text {ref }}\right| /\left|M_{i} \cup M_{\text {ref }}\right|, \quad M_{i}$ being the metabolites set of the generated draft network $i$ and $M_{\text {ref }}$ being the metabolites set of the reference network (manually curated model).

\section{Calculation of ratio}

The ratio $(R)$ between the coverage and the percentage of additional elements was calculated to assess how similar a particular draft network was to the manually curated reconstruction. We called $R_{g}, R_{n}$ and $R_{m}$ to the $R$ calculated in terms of genes, reactions, and metabolites, respectively. Thus, $R_{g}, R_{n}$ and $R_{m}$ were calculated as:

$R_{g}=\left|G_{i} \cap G_{r e f}\right| /\left|G_{i}-G_{r e f}\right|, G_{i}$ being the genes set of the generated draft network $i$ and $G_{\text {ref }}$ being the genes set of the reference network (manually curated model).

$R_{r}=\left|R_{i} \cap R_{r e f}\right| /\left|R_{i}-R_{r e f}\right|, R_{i}$ being the reactions set of the generated draft network $i$ and $R_{j}$ being the reactions set of the reference network (manually curated model).

$R_{m}=\left|M_{i} \cap M_{r e f}\right| /\left|M_{i}-M_{r e f}\right|, M_{i}$ being the metabolites set of the generated draft network $i$ and $M_{j}$ being the metabolites set of the reference network (manually curated model). 


\section{Evaluation of performance}

We created three models of Lactobacillus plantarum with CarveMe version 1.2.1 and ModelSEED version 2.4, using different media compositions for the gap-filling procedure that is carried out internally in these tools. Since the models were not able to generate biomass with the original media composition of CDM, PMM7, and PMM5 [43], we modified these mediums to ensure growth. The lack of growth was because of the presence of some compounds in the biomass equation which were not provided in the media. The modified mediums were called CMM-like, PMM7-like, PMM5-like, respectively (Additional file 1: File S2).

A set of 34 single-omission experiments [43] were used to evaluate the performance of the models. True positive were defined as growth in vivo and in silico; True negatives as no growth in vivo and in silico; False positives as no growth in vivo and growth in silico; False negatives as growth in vivo but no growth in silico. CDM-like media was used as a basal media for the single omission experiments. For both in vivo and in silico experiments, growth rates below $10 \%$ of the growth rate obtained in CDM-like were considered as no growth.

Metrics to evaluate the performance were calculated as follows:

$$
\begin{aligned}
& \text { Sensitivity }=\frac{\mathrm{TP}}{(\mathrm{TP}+\mathrm{FN})} \\
& \text { Specificity }=\frac{\mathrm{TN}}{(\mathrm{TN}+\mathrm{FP})} \\
& \text { Precision }=\frac{\mathrm{TP}}{(\mathrm{TP}+\mathrm{FP})} \\
& \text { Negative Predictive Value }(\mathrm{NPV})=\frac{\mathrm{TN}}{(\mathrm{TN}+\mathrm{FN})} \\
& \text { Accuracy }=\frac{\mathrm{TP}+\mathrm{TN}}{(\mathrm{TP}+\mathrm{TN}+\mathrm{FN}+\mathrm{FP})} \\
& \text { F score }=2 \times \frac{(\text { precision } \times \text { sensitivity })}{(\text { precision }+ \text { sensitivity })}
\end{aligned}
$$

\section{Availability}

All the reconstructions used as well as the MATLAB functions to generate the models (when possible) and to compare them are available at https:/github.com/SystemsBioinformatics/pub-data/tree/master/reconstructiontools-assessment [78]. In particular, the collection of plain text files showing examples of reactions in the manually curated models that were not recovered even though the associated genes were present in the draft reconstructions can be accessed in https://github.com/ SystemsBioinformatics/pub-data/tree/master/reconstruction- tools-assessment/supplementary\%20material/lpl and https:// github.com/SystemsBioinformatics/pub-data/tree/master/ reconstruction-tools-assessment/supplementary\%20material/bpe, for $L$. plantarum and B. pertussis, respectively. The code is distributed under a General Public License (GPL), an open-source license compliant with OSI (http:// opensource.org/licenses).

\section{Additional files}

Additional file 1: Supplementary figures, tables and files. (RAR $559 \mathrm{~kb}$ ) Additional file 2: Review history. (DOCX $2969 \mathrm{~kb}$ )

\section{Abbreviations}

FBA: Flux balance analysis; GSMM: Genome-scale metabolic model; JD: Jaccard distance; LAB: Lactic acid bacterium; R: Ratio between the coverage and the percentage of additional elements

\section{Acknowledgements}

S.N.M. acknowledges the financial support from CONICYT Becas Chile \#72180373 and Chr. Hansen. All the authors acknowledge the professional support of the specialist teams from each of the reconstruction platforms evaluated in this study.

\section{Review history}

The review history is available at Additional file 2.

\section{Authors' contributions}

BT and SNM conceived the project. All the authors designed the methodology. SNM generated the metabolic networks using the different reconstruction tools, wrote the code to compare them and evaluated the performance of the tools. All the authors contributed to the analysis of results. BT provided computing resources and supervised the research. SNM wrote the draft of the paper. All the authors read, edited, and approved the final version of the manuscript.

\section{Funding}

S.N.M acknowledges funding from CONICYT Becas Chile grant \#72180373 (https://www.conicyt.cl/becasconicyt/) and Chr. Hansen (https://www.chrhansen.com). B.T., B.G.O., and S.N.M. acknowledge funding for the ERACoBioTech project \#053.80.733 YogurtDesign (https://www.cobiotech.eu). The funders had no role in study design, data collection and analysis, decision to publish, or preparation of the manuscript.

\section{Availability of data and materials}

The datasets generated and/or analyzed during the current study are available in the GitHub repository, https://github.com/SystemsBioinformatics/pub-data/ tree/master/reconstruction-tools-assessment [78]. In particular, the collection of plain text files showing examples of reactions in the manually curated models that were not recovered even though the associated genes were present in the draft reconstructions can be accessed in https://github.com/

SystemsBioinformatics/pub-data/tree/master/reconstruction-tools-assessment/ supplementary\%20material//pl and https://github.com/SystemsBioinformatics/ pub-data/tree/master/reconstruction-tools-assessment/supplementary\%2 Omaterial/bpe [78], for L. plantarum and B. pertussis, respectively. The code is distributed under a General Public License (GPL), an open-source license compliant with OSI (http://opensource.org/licenses).

Ethics approval and consent to participate Not applicable.

Consent for publication

Not applicable

Competing interests

The authors declare that they have no competing interests. 


\section{Author details}

'Systems Bioinformatics, AIMMS, Vrije Universiteit Amsterdam, Amsterdam, The Netherlands. ${ }^{2}$ BioQUANT/COS, Heidelberg University, Heidelberg, Germany.

Received: 15 March 2019 Accepted: 22 July 2019

Published online: 07 August 2019

\section{References}

1. Øyås $\mathrm{O}$, Stelling J. Genome-scale metabolic networks in time and space. Curr Opin Syst Biol. 2017;8:51-8. https://doi.org/10.1016/j.coisb.2017.12.003.

2. Monk J, Nogales J, Palsson BO. Optimizing genome-scale network reconstructions. Nat Biotechnol. 2014;32:447-52. https://doi.org/10.1038/nbt.2870.

3. McCloskey D, Palsson B $\varnothing$, Feist AM. Basic and applied uses of genome-scale metabolic network reconstructions of Escherichia coli. Mol Syst Biol. 2013;9: 661. https://doi.org/10.1038/msb.2013.18.

4. Oberhardt MA, Palsson BØ, Papin JA. Applications of genome-scale metabolic reconstructions. Mol Syst Biol. 2009;5:320. https://doi.org/10.1038/ msb.2009.77.

5. Thiele I, Palsson $B \varnothing$. A protocol for generating a high-quality genome-scale metabolic reconstruction. Nat Protoc. 2010;5:93-121. https://doi.org/10.103 8/nprot.2009.203.

6. Francke C, Siezen RJ, Teusink B. Reconstructing the metabolic network of a bacterium from its genome. Trends Microbiol. 2005;13:550-8. https://doi. org/10.1016/j.tim.2005.09.001.

7. Feist AM, Herrgård MJ, Thiele I, Reed JL, Palsson B. Reconstruction of biochemical networks in microorganisms. Nat Rev Microbiol. 2009;7:129-43. https://doi.org/10.1038/nrmicro1949.

8. Hamilton JJ, Reed JL. Software platforms to facilitate reconstructing genome-scale metabolic networks. Environ Microbiol. 2014;16:49-59. https://doi.org/10.1111/1462-2920.12312.

9. Faria JP, Rocha M, Rocha I, Henry CS. Methods for automated genome-scale metabolic model reconstruction. Biochem Soc Trans. 2018. https://doi.org/1 0.1042/BST20170246

10. Kim WJ, Kim HU, Lee SY. Current state and applications of microbial genome-scale metabolic models. Curr Opin Syst Biol. 2017;2:10-8.

11. Teusink B, Wiersma A, Molenaar D, Francke C, de Vos WM, Siezen RJ, et al. Analysis of growth of Lactobacillus plantarum WCFS1 on a complex medium using a genome-scale metabolic model. J Biol Chem. 2006;281: 40041-8. https://doi.org/10.1074/jbc.M606263200.

12. Branco dos Santos F, Olivier BG, Boele J, Smessaert V, De Rop P, Krumpochova $\mathrm{P}$, et al. Probing the genome-scale metabolic landscape of Bordetella pertussis, the causative agent of whooping cough. Appl Environ Microbiol. 2017:83:e01528-17. https://doi.org/10.1128/AEM.01528-17.

13. Karlsen E, Schulz C, Almaas E. Automated generation of genome-scale metabolic draft reconstructions based on KEGG. BMC Bioinformatics. 2018; 19:467. https://doi.org/10.1186/s12859-018-2472-z.

14. Heirendt L, Arreckx S, Pfau T, Mendoza SN, Richelle A, Heinken A, et al. Creation and analysis of biochemical constraint-based models using the COBRA Toolbox v.3.0. Nat Protoc. 2019:1-64. https://doi.org/10.1038/s41596018-0098-2.

15. Aite M, Chevallier M, Frioux C, Trottier C, Got J, Cortés MP, et al. Traceability, reproducibility and wiki-exploration for "à-la-carte" reconstructions of genome-scale metabolic models. PLoS Comput Biol. 2018;14:1-25. https:// doi.org/10.1371/journal.pcbi.1006146.

16. Loira N, Zhukova A, Sherman DJ. Pantograph: a template-based method for genome-scale metabolic model reconstruction. J Bioinforma Comput Biol. 2015;13:1550006. https://doi.org/10.1142/S0219720015500067.

17. Caspi R, Billington R, Fulcher CA, Keseler IM, Kothari A, Krummenacker M, et al. The MetaCyc database of metabolic pathways and enzymes. Nucleic Acids Res. 2018;46. https://doi.org/10.1093/nar/gkx935.

18. King ZA, Lu J, Dräger A, Miller P, Federowicz S, Lerman JA, et al. BiGG models: a platform for integrating, standardizing and sharing genome-scale models. Nucleic Acids Res. 2016;44. https://doi.org/10.1093/nar/gkv1049.

19. Machado D, Andrejev S, Tramontano M, Patil KR. Fast automated reconstruction of genome-scale metabolic models for microbial species and communities Nucleic Acids Res. 2018;46:7542-53. https://doi.org/10.1093/nar/gky537.

20. Hanemaaijer M, Olivier BG, Röling WFM, Bruggeman FJ, Teusink B. Modelbased quantification of metabolic interactions from dynamic microbialcommunity data. PLoS One. 2017;12:e0173183. https://doi.org/10.1371/ journal.pone.0173183.
21. Olivier BG. MetaDraft [Internet]. Zenodo; 2018. doi:https://doi.org/10.5281/ zenodo.2398336.

22. Hucka M, Bergmann FT, Hoops S, Keating SM, Sahle S, Schaff JC, et al. The systems biology markup language (SBML): language specification for level 3 version 1 Core. J Integr Bioinform. 2017;12. https://doi.org/10.2390/biecolljib-2015-266.

23. Olivier BG, Bergmann FT. SBML level 3 package: flux balance constraints version 2. J Integr Bioinform. 2018;15:1-39. https://doi.org/10.1515/jib-2 017-0082.

24. Hucka M, Smith LP. SBML Level 3 package: Groups, Version 1 Release 1. J Integr Bioinform. 2017;13:290. https://doi.org/10.2390/biecoll-jib-2016-2 90.SBML.

25. Wang H, Marcišauskas S, Sánchez BJ, Domenzain I, Hermansson D, Agren R, et al. RAVEN 2.0: A versatile toolbox for metabolic network reconstruction and a case study on Streptomyces coelicolor. PLoS Comput Biol. 2018;14:117. https://doi.org/10.1371/journal.pcbi.1006541.

26. Kanehisa M, Goto S. KEGG: Kyoto encyclopedia of genes and genomes. Nucleic Acids Res. 2000;28:27-30. https://doi.org/10.1093/nar/28.1.27.

27. Henry CS, DeJongh M, Best AA, Frybarger PM, Linsay B, Stevens RL. High-throughput generation, optimization and analysis of genome-scale metabolic models. Nat Biotechnol. 2010;28:977-82. https://doi.org/10.103 8/nbt.1672.

28. Overbeek R, Olson R, Pusch GD, Olsen GJ, Davis JJ, Disz T, et al. The SEED and the Rapid Annotation of microbial genomes using Subsystems Technology (RAST). Nucleic Acids Res. 2014;42:206-14. https://doi.org/10.1 093/nar/gkt1226.

29. Karp PD, Latendresse M, Paley SM, Krummenacker M, Ong QD, Billington R, et al. Pathway tools version 19.0 update: software for pathway/genome informatics and systems biology. Brief Bioinform. 2016;17:877-90. https://doi org/10.1093/bib/bbv079.

30. Dias O, Rocha M, Ferreira EC, Rocha I. Reconstructing genome-scale metabolic models with merlin. Nucleic Acids Res. 2015;43:3899-910. https:// doi.org/10.1093/nar/gkv294.

31. Arkin AP, Cottingham RW, Henry CS, Harris NL, Stevens RL, Maslov S, et al. KBase: the United States department of energy systems biology knowledgebase. Nat Biotechnol. 2018:36:566-9. https://doi.org/10.1038/nbt.4163.

32. Pitkänen $E$, Jouhten $P$, Hou J, Syed MF, Blomberg P, Kludas J, et al. Comparative genome-scale reconstruction of gapless metabolic networks for present and ancestral species. PLoS Comput Biol. 2014;10. https://doi. org/10.1371/journal.pcbi.1003465.

33. Pabinger S, Snajder R, Hardiman T, Willi M, Dander A, Trajanoski Z. MEMOSys 2.0: an update of the bioinformatics database for genome-scale models and genomic data. Database. 2014;2014:2-7. https://doi.org/10.1093/database/ bau004.

34. Boele J, Olivier BG, Teusink B. FAME, the flux analysis and modeling environment. BMC Syst Biol. 2012;6:8. https://doi.org/10.1186/1752-0509-6-8.

35. Liao YC, Tsai MH, Chen FC, Hsiung CA. GEMSiRV: a software platform for GEnome-scale metabolic model simulation, reconstruction and visualization. Bioinformatics. 2012;28:1752-8. https:/doi.org/10.1093/bioinformatics/bts267.

36. Liao YC, Chen JCY, Tsai MH, Tang YH, Chen FC, Hsiung CA. MrBac: a web server for draft metabolic network reconstructions for bacteria. Bioeng Bugs. 2011;2:284-7. https://doi.org/10.4161/bbug.2.5.16113.

37. Cottret L, Chazalviel M, Gloaguen Y, Camenen E, Merlet B, Portais J, et al. MetExplore: collaborative edition and exploration of metabolic networks. 2018:46:495-502. https://doi.org/10.1093/nar/gky301.

38. Thorleifsson SG, Thiele I. rBioNet: A COBRA toolbox extension for reconstructing high-quality biochemical networks. Bioinformatics. 2011;27: 2009-10. https://doi.org/10.1093/bioinformatics/btr308.

39. Feng $X, X u Y$, Chen $Y$, Tang YJ. MicrobesFlux: a web platform for drafting metabolic models from the KEGG database. BMC Syst Biol. 2012;6:94. https://doi.org/10.1186/1752-0509-6-94.

40. Swainston N, Smallbone K, Mendes P, Kell D, Paton N. The SuBliMinaL toolbox: automating steps in the reconstruction of metabolic networks. J Integr Bioinform. 2011;8:186. https://doi.org/10.2390/biecoll-jib-2011-186.

41. Arakawa K, Yamada Y, Shinoda K, Nakayama Y, Tomita M. GEM system: automatic prototyping of cell-wide metabolic pathway models from genomes. BMC Bioinformatics. 2006;7:1-11. https://doi.org/10.1186/14 71-2105-7-168.

42. Teusink B, Van Enckevort FHJ, Francke C, Wiersma A, Wegkamp A, Smid EJ, et al. In silico reconstruction of the metabolic pathways of Lactobacillus plantarum: comparing predictions of nutrient requirements with those from 
growth experiments. Appl Environ Microbiol. 2005;71:7253-62. https://doi. org/10.1128/AEM.71.11.7253.

43. Wegkamp A, Teusink B, de Vos WM, Smid EJ. Development of a minimal growth medium for lactobacillus plantarum. Lett Appl Microbiol. 2010;50:5764. https://doi.org/10.1111/j.1472-765X.2009.02752.x.

44. Gonyar LA, Gelbach PE, Mcduffie DG, Koeppel AF, Chen Q, Lee G, et al. In vivo gene essentiality and metabolism in Bordetella pertussis. mSphere. 2019:4:114. https:/doi.org/10.1128/mSphere.00694-18.

45. Ponomarova O, Gabrielli N, Sévin DC, Mülleder M, Zirngibl K, Bulyha K, et al. Yeast creates a niche for symbiotic lactic acid bacteria through nitrogen overflow. Cell Syst. 2017;5:345-57. https://doi.org/10.1016/j.cels.2017.09.002.

46. Oberhardt MA, Puchałka J, Martins dos Santos VAP, Papin JA. Reconciliation of genome-scale metabolic reconstructions for comparative systems analysis. PLoS Comput Biol. 2011;7:e1001116. https://doi.org/10.1371/journal. pcbi.1001116.

47. Yuan Q, Huang T, Li P, Hao T, Li F, Ma H. Pathway-consensus approach to metabolic network reconstruction for Pseudomonas putida KT2440 by systematic comparison of Published Models. PLoS One. 2017;12:1-19. https:/doi.org/10.13 71/journal.pone.0169437.

48. Kumar VS, Maranas CD. GrowMatch: An automated method for reconciling in silico/in vivo growth predictions. PLoS Comput Biol. 2009;5:e1000308. https://doi.org/10.1371/journal.pcbi.1000308.

49. Hartleb D, Jarre F, Lercher MJ. Improved metabolic models for E. coli and mycoplasma genitalium from GlobalFit, an algorithm that simultaneously matches growth and non-growth data sets. PLoS Comput Biol. 2016;12:122. https://doi.org/10.1371/journal.pcbi.1005036.

50. Medlock GL, Papin JA. Guiding the refinement of biochemical knowledgebases with ensembles of metabolic networks and semisupervised learning. bioRxiv. 2018:1-14. https://doi.org/10.1101/460071.

51. Blazier AS, Papin JA. Reconciling high-throughput gene essentiality data with metabolic network reconstructions. bioRxiv, 2018:1-36. https://doi. org/10.1101/415448.

52. Lieven C, Beber ME, Olivier BG, Bergmann FT, Correia K, Diener C, et al. Memote: a community driven effort towards a standardized genome-scale metabolic model test suite. bioRxiv. 2018:1-26. https://doi.org/10.1101/35 0991.

53. Le Novère N, Finney A, Hucka M, Bhalla US, Campagne F, Collado-vides J, et al. Minimum information requested in the annotation of biochemical models (MIRIAM). Nat Biotechnol. 2005;23:1509-15. https://doi.org/10.1038/nbt1156.

54. Teusink B, Smid EJ. Modelling strategies for the industrial exploitation of lactic acid bacteria. Nat Rev Microbiol. 2006;4:46-56. https://doi.org/10.1038/ nrmicro1319.

55. Mujagic Z, De Vos P, Boekschoten MV, Govers C, Pieters HJHM, De Wit NJW, et al. The effects of Lactobacillus plantarum on small intestinal barrier function and mucosal gene transcription; a randomized double-blind placebo controlled trial. Sci Rep. 2017;7:1-11. https://doi.org/10.1038/srep40128.

56. Molin G. Probiotics in foods not containing milk or milk constituentes, with special reference to Lactobacillus plantarum 299v. Am J Clin Nutr. 2001;73: 380S-5S. https://doi.org/10.1093/ajcn/73.2.380s.

57. Heinken A, Thiele I. Anoxic conditions promote species-specific mutualism between gut microbes in silico. Appl Environ Microbiol. 2015;81:4049-61. https://doi.org/10.1128/AEM.00101-15.

58. Saulnier DM, Santos F, Roos S, Mistretta TA, Spinler JK, Molenaar D, et al. Exploring metabolic pathway reconstruction and genome-wide expression profiling in Lactobacillus reuteri to define functional probiotic features. PLoS One. 2011;6. https://doi.org/10.1371/journal.pone.0018783.

59. Melvin JA, Scheller EV, Miller JF, Cotter PA. Bordetella pertussis pathogenesis: current and future challenges. Nat Rev Microbiol. 2014;12: 274-88. https://doi.org/10.1038/nrmicro3235.

60. Schmid A, Dordick JS, Hauer B, Kiener A, Wubbolts M, Witholt B. Industrial biocatalysis today and tomorrow. Nature. 2001;409:258-68. https://doi.org/1 $0.1038 / 35051736$.

61. Magnúsdóttir S, Heinken A, Kutt L, Ravcheev DA, Bauer E, Noronha A, et al. Generation of genome-scale metabolic reconstructions for 773 members of the human gut microbiota. Nat Biotechnol. 2016;35:81-9. https://doi.org/1 $0.1038 /$ nbt.3703.

62. Moretti S, Martin O, Van Du Tran T, Bridge A, Morgat A, Pagni M. MetaNetX/ MNXref - reconciliation of metabolites and biochemical reactions to bring together genome-scale metabolic networks. Nucleic Acids Res. 2016;44: D523-6. https://doi.org/10.1093/nar/gkv1117.
63. Hahn AS, Altman T, Konwar KM, Hanson NW, Kim D, Relman DA, et al. A geographically-diverse collection of 418 human gut microbiome pathway genome databases. Sci Data. 2017;4:1-12. https://doi.org/10.103 8/sdata.2017.35.

64. Wilkinson MD, Dumontier M, Aalbersberg IJ, Appleton G, Axton M, Baak A, et al. The FAIR Guiding Principles for scientific data management and stewardship. Sci Data. 2016;3:1-9. https://doi.org/10.1038/sdata.2016.18.

65. Sánchez B, Nielsen J. Genome scale models of yeast: towards standardized evaluation and consistent omic integration. Integr Biol. 2015;7:846-58. https://doi.org/10.1039/c5ib00083a.

66. Monk JM, Lloyd CJ, Brunk E, Mih N, Sastry A, King Z, et al. iML1515, a knowledgebase that computes Escherichia coli traits. Nat Biotechnol. 2017;35:8-12.

67. Thiele I, Swainston N, Fleming RMT, Hoppe A, Sahoo S, Aurich MK, et al. A community-driven global reconstruction of human metabolism. Nat Biotechnol. 2013;31:419-25. https://doi.org/10.1038/nbt.2488.

68. Sauls JT, Buescher JM. Assimilating genome-scale metabolic reconstructions with modelBorgifier. Bioinformatics. 2014;30:1036-8. https://doi.org/10.1093/ bioinformatics/btt747.

69. Pham N, Van Heck RGA, Van Dam JCJ, Schaap PJ, Saccenti E, Suarez-diez M. Consistency, inconsistency, and ambiguity of metabolite names in biochemical databases used for genome-scale metabolic modelling. Metabolites. 2019;9:28. https://doi.org/10.3390/metabo9020028.

70. Opdam S, Richelle A, Kellman B, Li S, Daniel C, Lewis NE, et al. A systematic evaluation of methods for tailoring genome-scale metabolic models. Cell Syst. 2018:4:318-29. https://doi.org/10.1016/j.cels.2017.01.010.A.

71. Machado D, Herrga M. Systematic evaluation of methods for integration of transcriptomic data into constraint-based models of metabolism. PLoS Comput Biol. 2014;10: e1003580. https://doi.org/10.1371/journal.pcbi.1003580.

72. Siezen RJ, Francke C, Renckens B, Boekhorst J, Wels M, Kleerebezem M, et al. Complete resequencing and reannotation of the Lactobacillus plantarum WCFS1 genome. J Bacteriol. 2012;194:195-6. https://doi.org/1 0.1128/JB.06275-11.

73. Siezen RJ, Francke C, Renckens B, Boekhorst J, Wels M, Kleerebezem M, et al. Lactobacillus plantarum WCFS1, complete genome. NCBI. 2016; https:// www.ncbi.nlm.nih.gov/nuccore/NC_004567.2.

74. Parkhill J, Sebaihia M, Preston A, Murphy LD, Thomson N, Harris DE, et al. Comparative analysis of the genome sequences of Bordetella pertussis, Bordetella parapertussis and Bordetella bronchiseptica. Nat Genet. 2003;35: 32-40. https://doi.org/10.1038/ng1227.

75. Parkhill J, Sebaihia M, Preston A, Murphy LD, Thomson N, Harris DE, et al. Bordetella pertussis Tohama I, complete genome. NCBI. 2016; https:/www. ncbi.nlm.nih.gov/nuccore/NC_002929.2

76. Belda E, Van Heck RGA, Fraser C, Klenk H, Sekowska A, Vallenet D, et al. The revisited genome of Pseudomonas putida KT2440 enlightens its value as a robust metabolic chassis. Environ Microbiol. 2016;18:3403-24. https://doi. org/10.1111/1462-2920.13230.

77. Belda E, Van Heck RGA, Fraser C, Klenk H, Sekowska A, Vallenet D, et al. Pseudomonas putida KT2440 chromosome, complete genome. NCBI. 2016; https://www.ncbi.nlm.nih.gov/nuccore/NC_002947.4.

78. Mendoza SN, Olivier BG, Molenaar D, Teusink B, A systematic assessment of current genome-scale metabolic reconstruction tools. Data sets and source code. GitHub. 2019. https://github.com/SystemsBioinformatics/pub-data/ tree/master/reconstruction-tools-assessment.

\section{Publisher's Note}

Springer Nature remains neutral with regard to jurisdictional claims in published maps and institutional affiliations. 